\title{
Soft Sensing Based In Situ Control of Thermo-Fluidic Processes in DoD Inkjet Printing
}

\author{
Amritam Das*, Siep Weiland*
}

\begin{abstract}
This paper introduces a closed-loop control strategy for maintaining consistency of ink temperature in commercial Drop on Demand (DoD) inkjet printing. No additional sensors or additional actuators are installed in the printhead while achieving the consistency in ink temperature. To this end, this paper presents a novel in situ sensing-actuation policy at every individual ink-nozzle, where the jetting mechanism has three distinct roles. It is used for jetting liquid droplet onto the print media based on the print-job. It is used as a softsensor to estimate the real-time liquid temperature of the jetting nozzle. While not jetting liquid, it is used as a heating actuator to minimize the gradient of liquid temperature among jetting nozzles. The soft-sensing based in situ controller is implemented in an experimentally validated digital twin that models the thermo-fluidic processes of the printhead. The digital twin is scalable and flexible to incorporate an arbitrary number of inknozzles, making the control strategy applicable for future designs of the printhead.
\end{abstract}

Index Terms-Thermo-Fluidic Processes, Modeling, Soft Sensing, Optimal Control.

\section{INTRODUCTION}

Printing makes the human intellect tangible and timeless. From the early Gutenberg Press in the fifteenth century until today's printing technology, the documentation in print has enabled humanity to preserve its creativity in the form of books, crafts, and numerous consumable objects. Until the nineteenth century, human operators were employed to execute printing jobs manually using press machines [1]. During the early 1950s, the introduction of digital computers loomed the possibility of printing digital images on a medium without human intervention. The printing press was substituted by the digital inkjet technology in which liquid droplets of ink are deposited on a solid medium to replicate digital images in an automated fashion [2]. Since then, inkjet-based digital printing has been improved to be a faster, more cost-effective, and energy-efficient process. The non-contact and additive nature of inkjet printing has also attracted versatile applications. With the emergence of additive micro-manufacturing, inkjet printing is now applicable to a wide range of materials, including polymers and metals, and also various media like textile, wood, and circuit boards [3]. Thanks to the technical developments in inkjet-based digital printing, we are now gradually transcending 'from the world of paper printing to printing the world.'

\footnotetext{
${ }^{*}$ Corresponding author Amritam Das. Tel. +31(0)402472300. The authors are with the Control Systems Group, Department of Electrical Engineering, Eindhoven University of Technology, Eindhoven, The Netherlands. email: am.das@tue.nl, s.weiland@tue.nl
}

Today, the printer is a commercial peripheral which produces printed products based on the user's demand. Due to the constant increase in the market competition of inkjet printing, the print quality stands out to be a decisive factor. In general, the print quality indicates how accurate the printed product resembles its digital counterpart. However, the quantitative metric which determines the quality of the printed product depends mainly on the application area and the user group. For example, in paper printing, the measure of quality is typically determined by the reflectance of the printed area, print density variations, offsets of the printed pixels, etc. [4]. For textile printing, the measure of quality is the gloss of the printed surface, texture geometry, surface smoothness of the printed area, etc. [3].

As the application domain of inkjet printing is quite diverse, printer manufacturers concentrate on how to improve the general process of inkjet printing instead of considering any application-specific metric. To this end, the primary motivation behind this research is to study the role of liquid ink on improving the print quality irrespective of the printing medium and its application. One can view the inkjet printing process as a physical integration of liquid ink with a solid medium. This process is also known as jetting. Based on the user-defined print-job (a set of to-be-printed images), the jetting process deposits droplets of liquid on a solid medium (e.g., a sheet of paper). A set of printheads is responsible for generating and jetting the droplets in the right sequence, at the right position, with a pre-specified volume and with well-defined (or carefully controlled) physiochemical properties (such as viscosity, surface tension, temperature, etc.).

\section{A. General Architecture of Inkjet Printhead}

A common architecture of commercial printheads is shown in Figure 1a Here, the bottom stage of the printhead is called the nozzle-platform (NP), which consists of an array (divided into rows and columns) of nozzles. An example of the nozzles' placement with respect to an NP is shown in Figure 1b. The NP can be removed from the printhead and replaced with a new design. This feature allows for accommodating arbitrary numbers of nozzles without re-designing the entire printhead. On arrival of a print-job, the following aspects play key roles in the jetting process:

1) Mechanism for Drop Formation: Every individual nozzle comprises a conduit, an ink chamber, and a mechanism for ejecting ink from the chamber, through the conduit, onto a printing media. This jetting mechanism is equipped with a piezoelectric element attached to a diaphragm. Using a drive circuit, a specific voltage pulse is applied to the piezoelectric 


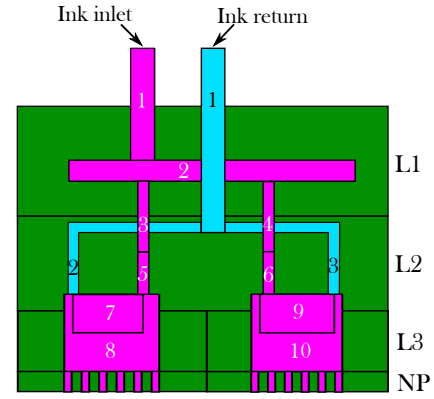

(a) Architecture of a printhead.

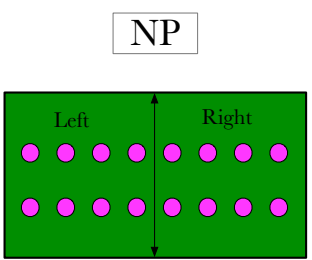

(b) Bottom-view of an NP with nozzles.

Fig. 1: The printhead consists of solid components (green), and channels for ink (inlet in purple and return in blue).

element to form a pressure wave inside the nozzle so as to create a droplet of liquid ink with pre-defined volume and push it out onto the medium [4](pp. 9-10).

2) Bit-map: The user-defined image is translated into a bit-map. A bit-map stores the sequence in which nozzles are actuated for drop formation in individual nozzles.

3) Ink Inlet and Ink Re-Circulation: Based on the image, the required flow rate for the deposition and re-circulation of ink is pre-determined and assigned for every individual nozzle.

\section{B. Quality Limiting Aspects in Inkjet Printhead: Thermo- Fluidic Processes}

For optimum print quality, maintaining a specific volume of ink, its surface tension, and its viscosity are essential. Furthermore, the temperature of ink droplets significantly affects them [5] (page 477). For example, change in viscosity, and surface tension often forms drop-satellites during jetting [6](Chapter 5) and harms the quality of the printed image. During the jetting process, temperature fluctuations of ink droplets among the nozzles may occur due to the following reasons:

1) Heat Dissipation in Nozzles: Jetting actuators dissipate heat while actuating (e.g. resistive property of piezoelectric material) [4] (page 2). Moreover, depending on drop volume, a jetting droplet results in impeding thermal energy. In particular, if not all the nozzles are simultaneously used for jetting, a gradient in ink temperature among jetting and non-jetting nozzles is evident.

2) Cross-talk in Solid and Liquid: In Figure 1b, the array of nozzles are physically coupled via the solid structure of the NP. Due to the different physical properties of solid and liquid, their mutual cross-talk will cause changes in temperature among nozzles as well as adjacent printheads.

The exchange of thermal energy (heat flux) on mutually interacting solid and liquid substances is a thermo-fluidic process. Concerning inkjet printing, the effect of this thermofluidic process is the temperature fluctuation of ink droplets among nozzles. Poor print quality is the immediate consequence of such fluctuation in ink temperature.

\section{State of The Art in Inkjet Technology}

In printing industry, model-based control of thermo-fluidic aspects is an unexplored field of research. The only litera- ture in this field are on physical modeling and experimental methods of drop formation and relevant fluid dynamics in inkjet printing. For example, [7] demonstrated the droplet formation of jetting process using an experimental setup. [2] performed physical modeling of the jetting process using conservation laws and validated the result using experimental study. [8] extended the model of jetting unit by including the model of piezoelectric actuators and their effects. However, these models are either ill-conditioned or computationally intractable for control and diagnosis purpose.

On the other hand, due to the development of faster and cheaper hardware, the scope of model-based automatic control has become viable in inkjet printing. However, designing control strategies to improve thermo-fludidic aspects is still rather unexplored domain. To the best of our knowledge, for the first time, [2] used a systems and control approach to tackle the operational issues in a jetting process. In this context, [4], [9] provides a holistic overview of the system theoretic approaches in the automatic control of jetting process. As a result, a system-theoretic outlook to model and control thermofluidic aspects in inkjet printing is largely missing.

\section{Contribution}

This paper introduces, for the first time, a model-based feedback controller for the inkjet printhead that uses no additional sensors and no additional actuators to compensate for the fluctuation of ink temperature among nozzles. The applicability of the control system is demonstrated with the help of three contributing aspects:

1) The modeling framework presents a digital twin of the printhead, which is modular towards an arbitrary number of nozzles. Change in the number of nozzles does not require redevelopment of the digital twin from the outset.

2) The piezoelectric elements that are already installed in every individual nozzle are used as collocated soft-sensors. The self-sensing capability of a piezoelectric element is exploited to develop a data-driven algorithm and estimate the ink temperature at every individual nozzle.

3) A concept of in situ actuation is introduced to control the fluctuation of ink temperature. Here, a sensing-actuation policy is introduced such that the controller uses the bitmap to anticipate the change in temperature at every 
nozzle and utilizes only the non-jetting nozzles to compensate for the temperature fluctuation.

\section{E. Organization of The Paper}

The remainder of this paper is organized as follows. After a brief explanation of the notations in Section II, the modeling framework is presented in Section III to design the digital twin. In Section IV, the developed digital twin is validated using an experimental set-up. In Section V, a soft-sensor is developed at every individual nozzle to estimate the ink temperature. A controller for compensating the fluctuation of ink temperature is developed and demonstrated in Section VI. Section VII provides some concluding remarks and directions for future research.

\section{NOTATIONS}

The set of natural numbers is denoted by $\mathbb{N}$. Given two positive integers $0<n<m$, the subset $\mathbb{N}_{[n, m]}:=\{n, n+$ $1, \cdots, m\} \subset \mathbb{N}$ denotes all natural number between $n$ and $m$ including $n, m$. The set of $n \times n$ positive semi-definite and positive definite matrices are denoted by $\mathbb{S}_{\succ 0}^{n}$ and $\mathbb{S}_{\succ 0}^{n}$, respectively. Let $a$ be a complex number defined as $a:=x+j y$ with $j=\sqrt{-1}$. Then, $\operatorname{Re}(a)$ denotes the real-part of $a$, i.e. $x$ and $\operatorname{Im}(a)$ denotes the imaginary-part of $a$, i.e. $y$. The complex conjugate of $a$ is defined by $a^{*}=x-j y$. Hermitian transpose of a $m \times n$ complex-valued matrix $A$ is defined by $A^{\mathrm{H}}$.

\section{A DIGITAL TWIN OF THERMO-FLUIDIC PROCESSES: PHYSICAL MODELING}

The first objective of the paper is to develop a digital twin of the jetting process for describing its thermo-fluidic aspects. The digital twin constitutes a generic physical model of the thermo-fluidic processes that can be used through out the design cycle. The digital twin must meet the following properties:

1) Modularity: The model is modular with arbitrary number of nozzles.

2) Flexibility: The model is flexible such that, during any stage of prototyping, necessary adaptation and modification on the model can be easily incorporated.

3) Versatility: The model serves as a versatile tool that can be used for simulation, design optimization, fault diagnosis, control and experimental validation.

To cope up with the ever increasing demands on through-put, the modularity of the digital twin offers the freedom of increasing the number of nozzles without the need of re-modeling. A flexible modeling approach can easily incorporate necessary adaptation at different stages of the design cycle, especially during experimental validation with increasing accuracy, and with supplementing additional functionality.

\section{A. A Graph Theoretic Modeling Framework}

This paper presents a graph theoretic framework that makes the digital twin modular, flexible and versatile. The graph theoretic model consists of the following three definitions:
D.1 Topology: A finite and connected graph consists of a set of nodes $\mathcal{N}$ and edges $\mathcal{E}$. It is defined by the following triad

$$
\mathcal{G}=(\mathcal{N}, \mathcal{E}, \mathbb{T}) .
$$

- The time axis is defined by set $\mathbb{T}$. If the thermo-fluidic processes are modeled in continuous time, $\mathbb{T}:=\mathbb{R}^{+}$. If discrete time is considered to model the thermofluidic processes, $\mathbb{T}:=\left\{k t_{d} \mid k \in \mathbb{N} \cup\{0\}\right\}$, where the sampling period $t_{d}$ is a fixed scalar. The time index is denoted by $t \in \mathbb{T}$.

- Each object $\mathcal{N}_{i}$ in the set $\mathcal{N}, i \in \mathbb{N}_{[1, m]}$, is a node or component of the graph.

- To realize the physical interconnection among nodes, an adjacency matrix $A \in \mathbb{N}^{m \times m}$ has either zero or one as its elements depending on whether the nodes $\mathcal{N}_{i}$ and $\mathcal{N}_{j}$ are physically attached or not. Precisely, its entries

$$
A_{i, j}= \begin{cases}1, & \text { if } \mathcal{N}_{i} \text { is connected to } \mathcal{N}_{j} \\ 0, & \text { otherwise. }\end{cases}
$$

- The set of edges $\mathcal{E}=\left\{\mathcal{E}_{i, j} \mid\right.$ for all $(i, j)$ with $A_{i, j}=$ $1\}$ describes the interconnection between a particular node and its neighbourhood nodes.

D.2 Edges: Every individual edge $\mathcal{E}_{i, j} \in \mathcal{E}$ is defined by the following couple:

$$
\mathcal{E}_{i, j}=\left(\mathcal{L}_{i, j}^{I}, \mathcal{M}_{i, j}\right) .
$$

- Wherever $\mathcal{N}_{i}$ is interconnected to $\mathcal{N}_{j}$, i.e. $A_{i, j}=1$, there are time-varying interconnection signals associated with the edge. Let these signals be $\left(l_{i, j}, l_{j, i}\right)$ : $\mathbb{T} \rightarrow \mathcal{L}_{i, j}^{I}$ respectively.

- The relation among interconnection signals $l_{i, j}$ and $l_{j, i}$ defines a subspace $\mathcal{M}_{i, j} \subset \mathcal{L}_{i, j}^{I}$.

D.3 Nodes: Every individual node $\mathcal{N}_{i}$ is associated with the following triple:

$$
\mathcal{N}_{i}=\left(\mathcal{S}_{i}, \mathcal{B}_{i}, \mathcal{P}_{i}\right)
$$

- There are three categories of time-varying signals associated with every individual $\mathcal{N}_{i}$.

(a) The internal state variable $x_{i}: \mathbb{T} \rightarrow \mathcal{X}_{i}$

(b) The to-be-manipulated control signals $c_{i}: \mathbb{T} \rightarrow \mathcal{C}_{i}$.

(c) Associated with every individual node, the collection of all interconnection signals is $l_{i}: \mathbb{T} \rightarrow \mathcal{L}_{i}$. They are related to an individual edge such that $\mathcal{L}_{i, j}^{I} \subseteq \mathcal{L}_{i} \times \mathcal{L}_{j}$.

(d) The user-defined print-job introduces a set of signals that affect the node behaviour. They are $d_{i}$ : $\mathbb{T} \rightarrow \mathcal{D}_{i}$.

As a result, $\mathcal{S}_{i}:=\mathcal{X}_{i} \times \mathcal{C}_{i} \times \mathcal{L}_{i} \times \mathcal{D}_{i}$.

- Print-job signals $d_{i}$ always depend on the user-defined image that is captured in a bit-map. Given a specific bit-map, the signals $d_{i}$ are restricted by a subspace $\mathcal{B}_{i} \subset \mathcal{D}_{i}$.

- The behaviour of $\mathcal{N}_{i}$ is captured by a relation among all the signals $\left(x_{i}, c_{i}, l_{i}, d_{i}\right)$ and defines a subspace $\mathcal{P}_{i} \subset$ $\mathcal{S}_{i}$. 


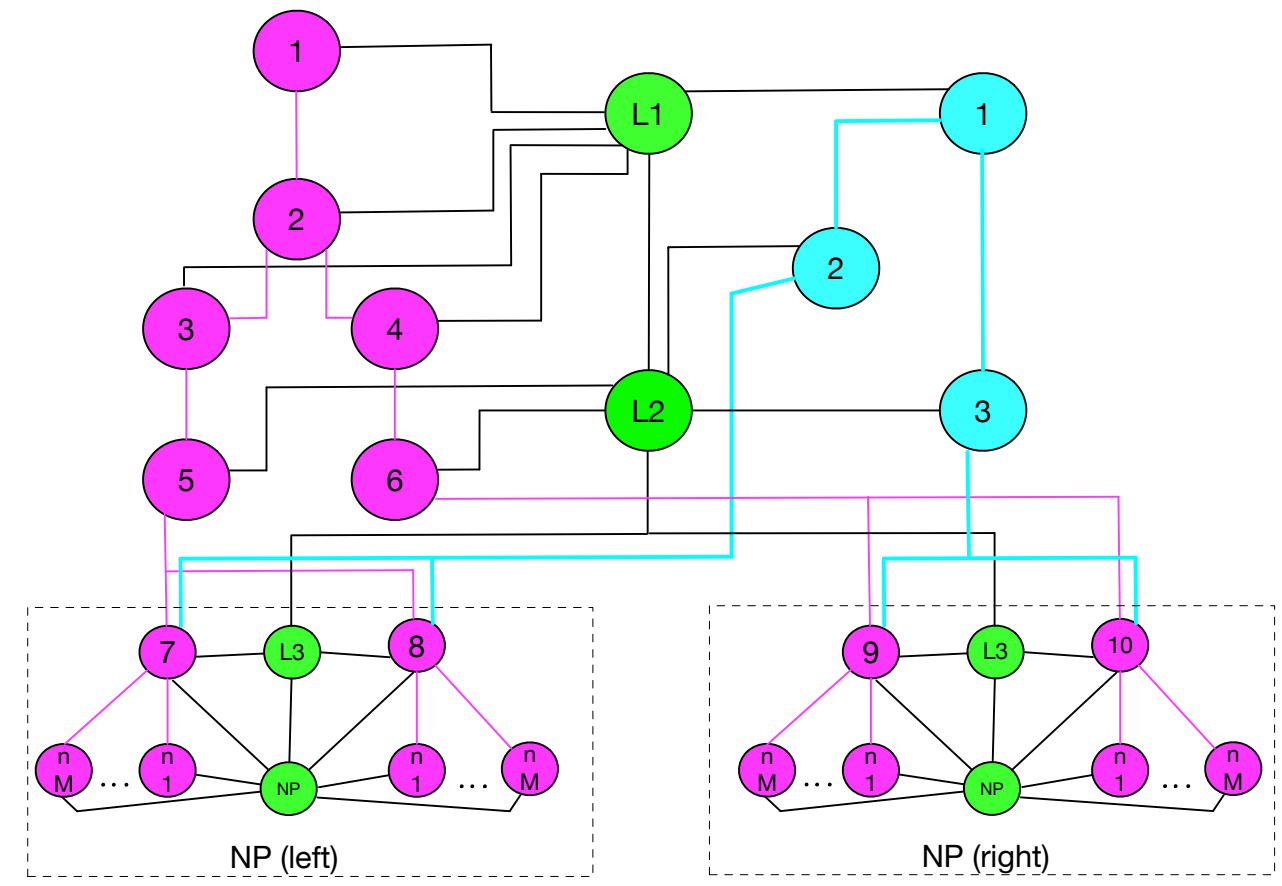

Fig. 2: Topology of the printhead with $2 \mathrm{M}$ nozzles (denoted by $n 1, \cdots, n M)$ ). Nozzles are equally divided over the left and right side of the NP. $\bigcirc$ represents a solid component, $\bigcirc$ represents an inlet channel for the ink, and $\bigcirc$ represents a return channel for the ink. The exchange of thermal energy (conduction) between two solid components or a solid and liquid component is represented by $\ldots$. The exchange of thermal energy (convection) due to inflow of ink along the inlet channels is represented by - The exchange of thermal energy (convection) due to return of ink along the ink return channels is represented by

\section{B. Graph Theoretic Digital Twin for Thermo-Fluidic Pro- cesses}

To adapt (D.1)-(D.3) towards the thermo-fluidic processes of a generic printhead, the user must specify the following items:

1) Printhead Topology: The digital twin of the printhead is a finite and connected graph according to the definition (D.1).

- Each node represents a solid or liquid component. For a specific design, the user specifies the number of nodes.

- The adjacency matrix $A$ is defined based on the architecture of the printhead.

- Except the adjustable NPs, the remaining architecture of the printhead is typically kept identical. Then, specifying the arrays of nozzles is sufficient for defining the topology of a newly designed printhead.

- The user defines $\mathbb{T}$ to specify whether the signals are in continuous time or discrete time.

For the printhead shown in figure $1 \mathrm{a}$, individual nodes (components) and the interconnection topology, is depicted in figure 2

2) Interconnection Among Components: The interconnection describes the exchange of thermal energy among adjacent components. The energy exchange is either conductive (between two solid components) or convective (between a solid and liquid component or between two liquid components). Typically, a bidirectional exchange of thermal energy occurs between two solid or a solid and a liquid component. A unidirectional exchange of thermal energy occurs between two adjacent liquid channels towards the direction of the flow. Hence, distinction is made between an edge $\mathcal{E}_{i, j}$ and an another edge $\mathcal{E}_{j, i}$ to distinguish between bidirectional and unidirectional interconnections.

To model individual edge between adjacent components $\mathcal{N}_{i}$ and $\mathcal{N}_{j}$, i.e. wherever $A_{i, j}=1$, the user must specify following items according to (D.2):

- Interconnection signals describe the exchange of thermal energy among adjacent components. In terms of temperature of the adjacent nodes $\left({ }^{\circ} \mathrm{C}\right)$, the interconnection signals between $\mathcal{N}_{i}$ and $\mathcal{N}_{j}$ are categorised into inputs $v_{i, j}(t) \in \mathbb{R}^{n_{v}^{i j}}$ and outputs $w_{i, j}(t) \in \mathbb{R}^{n_{w}^{i j}}$. Here, the user specifies the dimension of the interconnection signal $l_{i, j}(t)$, where $l_{i, j}:=\operatorname{col}\left(v_{i, j}, w_{i, j}\right)$.

- Based on the conductive or convective thermal energy exchange, the subspace $\mathcal{M}_{i, j}$ defines either uni-direction or bi-direction interconnection relation according to

$$
\mathcal{M}_{i, j}:=\left\{\left(l_{i, j}, l_{j, i}\right) \mid v_{j, i}=M_{i, j} w_{i, j} \wedge A_{i, j}=1\right\} .
$$

Wherever $A_{i, j}=1$, the user specifies the relation (4) by defining a constant matrix $M_{i, j}$ of suitable dimension. The matrix $M_{i, j}$ defines the weights associated to interconnection input-output pair individually.

3) Solid or Liquid Components: For individual node, based on the physical properties and dimensions, the Biot number [10]-[12] justifies the possibility to consider a lumped model (finite dimensional) while neglecting the spatial variation of thermo-fluidic aspects component. A lumped model describes 
the evolution of temperature at individual nodes due to a) the conduction between two solid components, b) convection between two liquid components, c) convection between a solid and a liquid component.

To model individual node $\mathcal{N}_{i}$, the user must specify the following items according to (D.3):

- The user specifies the dimension of the internal state variable $x_{i}(t) \in \mathbb{R}^{n_{x}^{i}}$ for individual node. In this case, $x_{i}$ is the temperature $\left({ }^{\circ} \mathrm{C}\right)$ of individual solid or liquid component.

- Control signals are categorised into control inputs (in terms of thermal power (Watt) that can be manipulated) $u_{i}(t) \in \mathbb{R}^{n_{u}^{i}}$ and measured outputs (in terms of temperature $\left({ }^{\circ} \mathrm{C}\right)$ that can be measured) $y_{i}(t) \in \mathbb{R}^{n_{y}^{i}}$. Here, the user specifies the dimension of the control signals $c_{i}(t)$, where $c_{i}:=\operatorname{col}\left(u_{i}, y_{i}\right)$.

- Stacking all the edges corresponding to an individual node, interconnection inputs $v_{i}(t) \in \mathbb{R}^{n_{v}^{i}}$ and outputs $w_{i}(t) \in \mathbb{R}^{n_{w}^{i}}$ are obtained. Based on the user-defined relation (4), these signals are constructed according to $v_{i}:=\operatorname{col}\left(v_{i, k}\right)_{k \in \mathbb{I}_{i, j}}$ and $w_{i}:=\operatorname{col}\left(w_{i, k}\right)_{k \in \mathbb{I}_{i, j}}$ with $\mathbb{I}_{i, j}=\left\{j \mid A_{i, j}=1\right\}$. Therefore, by stacking edges, the interconnection signals $l_{i}:=\operatorname{col}\left(v_{i}, w_{i}\right)$ are automatically defined for an indvidual node.

- Using an user-defined bit-map, the flow parameters of inlet ink and ink return $\left(\mathrm{m}^{3} / \mathrm{sec}\right)$ are known and stored in a time-varying matrix $\Theta(t)$ for all liquid channels. To allocate flow parameters for every individual node, an operator $\mathcal{T}_{i}$ is defined such that $\Theta_{i}(t)=\left(\mathcal{T}_{i}(\Theta)\right)(t)$, where $\Theta_{i}(t)$ is the allocated flow parameter for the node $\mathcal{N}_{i}$.

The matrix $\Theta_{i}(t)$ relates print-job associated inputs (in terms of convective heat flux, Watt) $p_{i}(t) \in \mathbb{R}^{n_{p}^{i}}$ and outputs $q_{i}(t) \in \mathbb{R}^{n_{q}^{i}}$ (in terms of thermal energy over unit volume of liquid, $\mathrm{Kg}^{\circ} \mathrm{C} / \mathrm{m}^{3}$ ) according to

$$
\mathcal{B}_{i}:=\left\{d_{i}=\operatorname{col}\left(p_{i}, q_{i}\right) \mid p_{i}=\Theta_{i} q_{i}\right\} .
$$

Here, 5) describes convective thermal energy transfer due to the change in the flow of ink inlet and ink return for every individual node. Using user-defined $\Theta(t)$ and $\mathcal{T}_{i}$ in (5), the signals $d_{i}(t)$ and the matrix $\Theta_{i}(t)$ are automatically defined for individual node.

- Depending on whether continuous time or discrete time is chosen for modeling, the dynamic relations among defined signals $\left(x_{i}, c_{i}, l_{i}, d_{i}\right)$ has the following inputstate-output form:

$$
\begin{gathered}
{\left[\begin{array}{c}
Q_{t}\left(x_{i}\right)(t) \\
w_{i}(t) \\
q_{i}(t) \\
y_{i}(t)
\end{array}\right]=} \\
{\left[\begin{array}{llll}
A_{x x}^{i} & B_{x v}^{i} & B_{x p}^{i} & B_{x u}^{i} \\
C_{w x}^{i} & D_{w v}^{i} & D_{w p}^{i} & D_{w u}^{i} \\
C_{q x}^{i} & D_{q v}^{i} & D_{q p}^{i} & D_{q u}^{i} \\
C_{y x}^{i} & D_{y v}^{i} & D_{y p}^{i} & D_{y u}^{i}
\end{array}\right]\left[\begin{array}{c}
x_{i}(t) \\
v_{i}(t) \\
p_{i}(t) \\
u_{i}(t)
\end{array}\right]} \\
+\left[\begin{array}{c}
f_{x}^{i}(t) \\
g_{w}^{i}(t) \\
g_{q}^{i}(t) \\
g_{y}^{i}(t)
\end{array}\right] .
\end{gathered}
$$

Equation (6) is derived using the laws of mass and energy balance (c.f. [13]). In practice, such physical modeling typically involves quantities that are continuous in time. If the user requires the model to be in discrete time, Based on the user's choice of $\mathbb{T}$, (6) can be used in either continuous time or discrete time. Accordingly, on functions $f: \mathbb{T} \rightarrow \mathbb{R}^{n}$, the operator $Q_{t}$ is defined as

$$
\left(Q_{t}(f)\right)(t):= \begin{cases}\frac{\mathrm{d} f(t)}{\mathrm{d} t}, & \text { if } t \in \mathbb{R}^{+}, \\ f\left(t+t_{d}\right), & \text { if } t \in\left\{k t_{d} \mid k \in \mathbb{N} \cup\{0\}\right\} .\end{cases}
$$

Consequently, the definitions of constant matrices $A_{x x}^{i}$, $B_{x v}^{i}, B_{x p}^{i}, B_{x u}^{i}$, and the function $f_{x}^{i}$ differ depending on the choice of $\mathbb{T}$. Precisely,

(a) In continuous time, $A_{x x}^{i}=A_{c x}^{i}, B_{x v}^{i}=B_{c v}^{i}, B_{x p}^{i}=$ $B_{c p}^{i}, B_{x u}^{i}=B_{c u}^{i}, f_{x}^{i}=f_{c x}^{i}$.

(b) Discretization of continuous time model, e.g. using Euler's forward method (c.f. [14]), yields $A_{x x}^{i}=$ $I+t_{d} A_{c x}^{i}, B_{x v}^{i}=t_{d} B_{c v}^{i}, B_{x p}^{i}=t_{d} B_{c p}^{i}, B_{x u}^{i}=t_{d} B_{c u}^{i}$, $f_{x}^{i}=t_{d} f_{c x}^{i}$.

Based on the material properties, chemical composition of the ink and physical dimension of individual component in the printhead, the matrices $A_{c, x}^{i}, B_{c, v}^{i}, B_{c, p}^{i}$, and $B_{c, u}^{i}$ are determined from the balance equations. They are real-valued constant matrices with suitable dimensions. The constant matrices $C_{m, n}^{i}$ and $D_{m, n}^{i}$ for $m, n \in$ $\{x, v, w, p, q, u, y\}$ are suitably defined based on the topology, the availability of sensors and control actuators. The functions $f_{c x}^{i}, g_{w}^{i}, g_{q}^{i}, g_{y}^{i}$ of suitable dimensions are introduced to model disturbances related to ambient temperature, convective losses due to movement of the carriage during printing, heating of the electronics etc.

Illustrative Example III.1. The complete steps for constructing the physical model are provided in the supplementary material attached in the Appendix.

\section{Equivalent Classes of Thermo-Fluidic Model}

In the previous exposition, the graph-theoretic representation (D.1)-(D.3) presents a paradigm for building and upscaling thermo-fluidic model for a new design of printhead with arbitrary number of nozzles. Subsequently, a compact multi-input and multi-output (MIMO) representation may appear to be useful for design optimization or synthesizing observer-based controller. By rearranging signals and performing algebraic operations, there are three ways to represent the graph theoretic model.

\section{1) By Stacking Node Signals:}

$$
\begin{aligned}
& \overline{\mathcal{P}}_{\mathrm{I}}:= \\
& \begin{array}{c}
(x, c, l, d) \text { such that, } \\
l \in\{l \mid v=\bar{M} w\}, \\
\left.\left[\begin{array}{c}
Q_{t}(x) \\
w \\
q \\
y
\end{array}\right]=\left[\begin{array}{llll}
A_{x x} & B_{x v} & B_{x p} & B_{x u} \\
C_{w x} & D_{w v} & D_{w p} & D_{w u} \\
C_{q x} & D_{q v} & D_{q p} & D_{q u} \\
C_{y x} & D_{y v} & D_{y p} & D_{y u}
\end{array}\right]\left[\begin{array}{l}
x \\
v \\
p \\
u
\end{array}\right]+\left[\begin{array}{c}
f_{x} \\
g_{w} \\
g_{q} \\
g_{y}
\end{array}\right]\right\}
\end{array}
\end{aligned}
$$




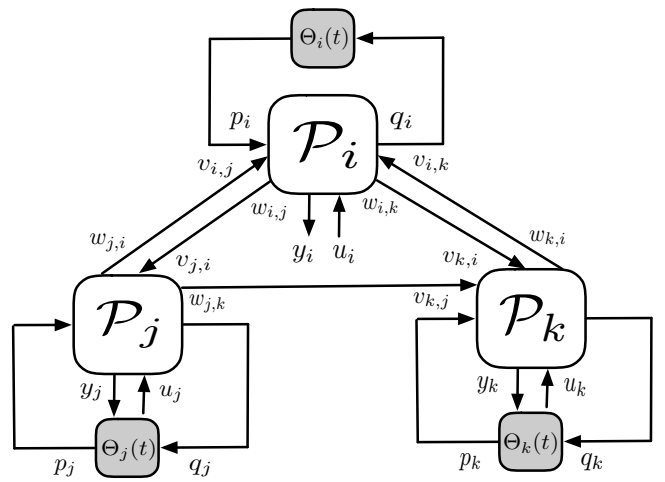

(a) Graph theoretic representation as defined by (D.1)-(D.3).

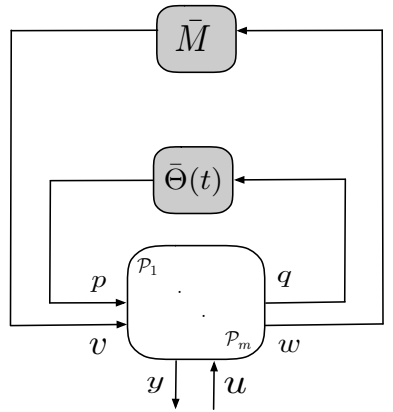

(b) Representation of $\overline{\mathcal{P}}_{\mathrm{I}}$ in (7)

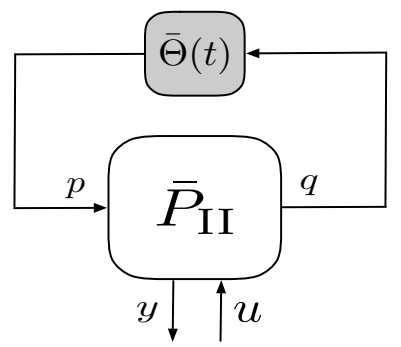

(c) Representation of $\overline{\mathcal{P}}_{\text {II }}$ in 8

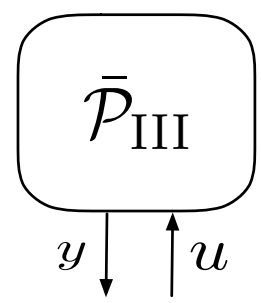

(d) Representation of $\overline{\mathcal{P}}_{\text {III }}$ in 9]

Fig. 3: Four equivalent representations of the thermo-fluidic model in the printhead.

\section{2) By Eliminating Interconnection Signals:}

$$
\overline{\mathcal{P}}_{\text {II }}:=
$$$$
\left\{\begin{array}{c}
(x, c, d) \text { such that, } \\
d \in\{d \mid p=\bar{\Theta} q\} \\
\left.\left[\begin{array}{c}
Q_{t}(x)(t) \\
q(t) \\
y(t)
\end{array}\right]=\left[\begin{array}{ccc}
A & B_{1} & B_{2} \\
C_{1} & D_{11} & D_{12} \\
C_{2} & D_{21} & D_{22}
\end{array}\right]\left[\begin{array}{l}
x(t) \\
p(t) \\
u(t)
\end{array}\right]+\left[\begin{array}{c}
f(t) \\
g_{1}(t) \\
g_{2}(t)
\end{array}\right]\right\}
\end{array}\right.
$$

\section{3) By Eliminating Print-Job Signals:}

$$
\begin{aligned}
& \overline{\mathcal{P}}_{\text {III }}:= \\
& \left\{\left[\begin{array}{c}
\left(Q_{t}(x)\right)(t) \\
y(t)
\end{array}\right]=\left[\begin{array}{ll}
(x, c) \text { such that, } \\
\tilde{A}(t) & \tilde{B}(t) \\
\tilde{C}(t) & \tilde{D}(t)
\end{array}\right]\left[\begin{array}{l}
x(t) \\
u(t)
\end{array}\right]+\left[\begin{array}{c}
G(t) \\
J(t)
\end{array}\right]\right\} .
\end{aligned}
$$

All four representations of the thermo-fluidic model are depicted in figure 3 . Among them, behaviour of the graph theoretic model (D.1)-(D.3) is equivalently described by $\overline{\mathcal{P}}_{\mathrm{I}}$ in (7). The equivalence holds as (7) is a result of signal re-arrangement in (D.1)-(D.3). In particular, $(x, c, l, d)$ are obtained by stacking the signals $\left(x_{i}, c_{i}, l_{i}, d_{i}\right)$ in a column for all $i \in \mathbb{N}_{[1, m]}$. In (7), the matrix $\bar{M}$ is constructed from the matrix $M_{i, j}$ by stacking the relation $v_{j, i}=M_{i, j} w_{i, j}$ whenever $A_{i, j}=1$ for all $i, j \in \mathbb{N}_{[1, m]}$. Similarly, the matrix $\bar{\Theta}(t)=\operatorname{diag}\left(\Theta_{i}(t)\right)_{i \in \mathbb{N}_{[1, m]}}$. The functions $f_{x}, g_{w}, g_{q}, g_{y}$ are obtained by stacking $f_{x}^{i}, g_{w}^{i}, g_{q}^{i}, g_{y}^{i}$ in a column over all $i \in \mathbb{N}_{[1, m]}$. Furthermore, for each $m, n \in\{x, v, w, p, q, u, y\}$, $A_{m n}, B_{m n}, C_{m n}, D_{m n}$ are obtained by diagonally stacking $A_{m n}^{i}, B_{m n}^{i}, C_{m n}^{i}, D_{m n}^{i}$ over all $i \in \mathbb{N}_{[1, m]}$.
On the other hand, obtaining behaviourally equivalent representation (8) and (9) from (7) is subject to satisfying the following conditions:

Theorem III.1. (Equivalence Under Signal Elimination)

1) $\overline{\mathcal{P}}_{\mathrm{II}}=\left\{(x, c, d) \mid \exists(x, c, l, d)\right.$ such that $\left.(x, c, l, d) \in \overline{\mathcal{P}}_{\mathrm{I}}\right\}$ if and only if $\left(I-D_{w v} \bar{M}\right)$ is invertible. Then, in (8),

$$
\begin{gathered}
{\left[\begin{array}{ccc}
A & B_{1} & B_{2} \\
C_{1} & D_{11} & D_{12} \\
C_{2} & D_{21} & D_{22}
\end{array}\right]=\left[\begin{array}{ccc}
A_{x x} & B_{x p} & B_{x u} \\
C_{q x} & D_{q p} & D_{q u} \\
C_{y x} & D_{y p} & D_{y u}
\end{array}\right]} \\
+\left[\begin{array}{c}
B_{x v} \\
D_{q v} \\
D_{y v}
\end{array}\right] \bar{M}\left(I-D_{w v} \bar{M}\right)^{-1}\left[\begin{array}{c}
C_{w x}^{\top} \\
D_{w p}^{\top} \\
D_{w u}^{\top}
\end{array}\right]^{\top},
\end{gathered}
$$

$$
\left[\begin{array}{c}
f(t) \\
g_{1}(t) \\
g_{2}(t)
\end{array}\right]=\left[\begin{array}{c}
B_{x v} \\
D_{q v} \\
D_{y v}
\end{array}\right] \bar{M}\left(I-D_{w v} \bar{M}\right)^{-1}\left[\begin{array}{c}
f_{x}^{\top}(t) \\
g_{q}^{\top}(t) \\
g_{y}^{\top}(t)
\end{array}\right]^{\top} \text {. }
$$

2) $\overline{\mathcal{P}}_{\text {III }}=\left\{(x, c) \mid \exists(x, c, d)\right.$ such that $\left.(x, c, d) \in \overline{\mathcal{P}}_{\text {II }}\right\}=$ $\left\{(x, c) \mid \exists(x, c, l, d)\right.$ such that $\left.(x, c, l, d) \in \overline{\mathcal{P}}_{\mathrm{I}}\right\}$ if and only if $\left(I-D_{11} \bar{\Theta}(t)\right)$ is invertible for all $t \in \mathbb{T}$. Then, in (9),

$$
\begin{aligned}
& {\left[\begin{array}{cc}
\tilde{A}(t) & \tilde{B}(t) \\
\tilde{C}(t) & \tilde{D}(t)
\end{array}\right]=\left[\begin{array}{cc}
A & B_{2} \\
C_{2} & D_{22}
\end{array}\right]} \\
& +\left[\begin{array}{c}
B_{1} \\
D_{21}
\end{array}\right] \bar{\Theta}(t)\left(I-D_{11} \Theta(t)\right)^{-1}\left[\begin{array}{c}
C_{1}^{\top} \\
D_{12}^{\top}
\end{array}\right]^{\top},
\end{aligned}
$$




$$
\left[\begin{array}{c}
G(t) \\
J(t)
\end{array}\right]=\left[\begin{array}{c}
B_{1} \\
D_{21}
\end{array}\right] \bar{\Theta}(t)\left(I-D_{11} \Theta(t)\right)^{-1}\left[\begin{array}{c}
f^{\top}(t) \\
g_{2}^{\top}(t)
\end{array}\right]^{\top}
$$

Remark III.1. (Assessment of the digital twin) The digital twin provides a modular modeling framework that is easily adjustable for arbitrary number of nozzles. In fact, one simply requires to specify the number nozzles and how they are distributed over rows. The digital twin then automatically build the model of every individual nozzle due to their identical dimension and physical properties. And the graph theoretic approach leverages an easy re-adjustment of nozzles with the rest of the printhead components.

Moreover, lumping individual solid and liquid component may not always provide sufficient accuracy to the developed model. In this case, the digital twin is built to be flexible towards further partitioning individual component into multiple sub-components. Taking a specific node from the graph, it can be partitioned into smaller identical nodes. Once the required model accuracy is achieved by partitioning, they are reconnected to the graph.

The versatile digital twin offers four equivalent representations of the thermo-fluidic model if the conditions in theorem III.1 are satisfied. Any one of these four representations can be used for design optimization of individual components, monitoring and fault diagnosis of nozzles, controller synthesis and deployment. However, the user's choice of a specific representation often depends on the hardware architecture, purpose of the model and time budget.

\section{EXPERIMENTAL VALIDATION OF THERMO-FLUIDIC MODEL}

\section{A. Experimental Setup}

For validation, an experimental set-up is built as shown in figure 4. The setup consists of two liquid vessels that are connected with a printhead that has 160 nozzles. These connections are of two types. The first connection relies on the siphoning effect, where the liquid flows from a higher placed vessel through the printhead towards a lower placed vessel. The level of both vessels is controlled by a pump that pushes the liquid from the lower vessel back to the upper vessel. Keeping those vessels at a constant level, a constant re-circulation flow through the printhead is established. To raise the temperature of the ink inlet in the printhead, the liquid in upper vessel heated up with a heater. In the NP of the printhead, two locations are selected to place NTC thermistors (in figure 5 they are called $T_{\text {left }}, T_{\text {right }}$ ) to receive real-time information of its temperature. They are adjacent to two specific nozzles. Among these two nozzles, one is located closer to the right-end of the NP and the other one is located closer to its left-end. In this way, both temperature evolution on the both side of the printhead can be monitored.

\section{B. Experiment Design}

An experiment is performed by operating the printhead on the printing of $3500 \mathrm{~A}-4$ sheets of paper in the following sequence:

1) For 100 seconds, first 500 pages are empty

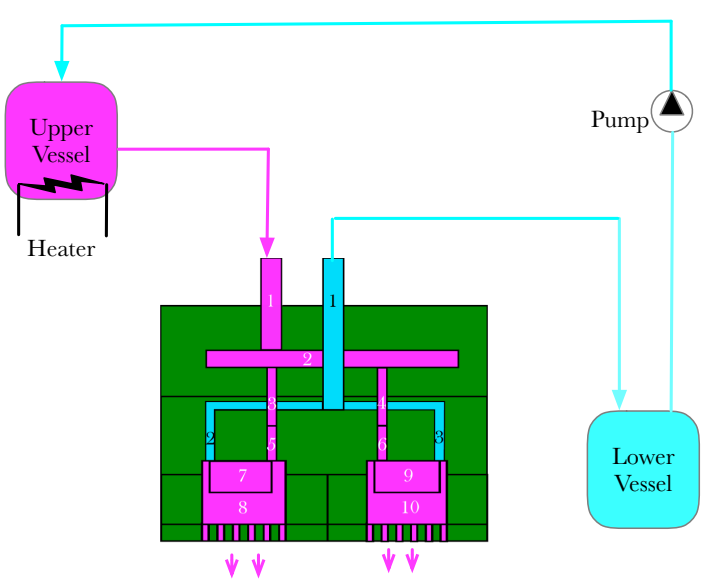

Fig. 4: Experimental Setup

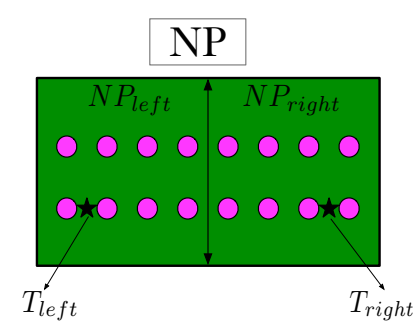

Fig. 5: Bottom view of NP. $\star$ denotes the locations of NTC thermistors.

2) In the next 100 seconds, another 500 pages are entirely printed with black ink by using all nozzles on left side of the NP $N P_{l e f t}$.

3) In the next 100 seconds, another 500 pages are empty.

4) In the next 100 seconds, another 500 pages are entirely printed with black ink by using half of the nozzles that are located on the left side of $N P_{\text {left }}$.

5) In the next 100 seconds, 500 pages are empty.

6) In the next 100 seconds, another 500 pages are entirely printed with black ink by using half of the nozzles that are located on the right side of $N P_{\text {left }}$.

7) In the next 100 seconds, the last 500 pages are empty.

\section{Setting Up The Digital Twin for Simulation}

Based on the above print-job, the bit-map is generated. Corresponding flow are assigned with the print-job signals. With 60 nozzles, in total, there are 186 nodes. Every individual node is associated with its temperature (in ${ }^{\circ} \mathrm{C}$ ) as its internal state. Based on the topology of the printhead (as shown in figure 2), the interconnection signals and their relations are built. The print-job also assigns the flow parameters to every individual node in terms the print-job signals and their relations. Once the model is built using the definitions (D.1)(D.3), any one of the four representations can be used for simulating the digital twin.

\section{Results}

In figure 6, at location $T_{\text {left }}$ and $T_{\text {right }}$, the temperature data measured by NTC thermistors are compared against the 


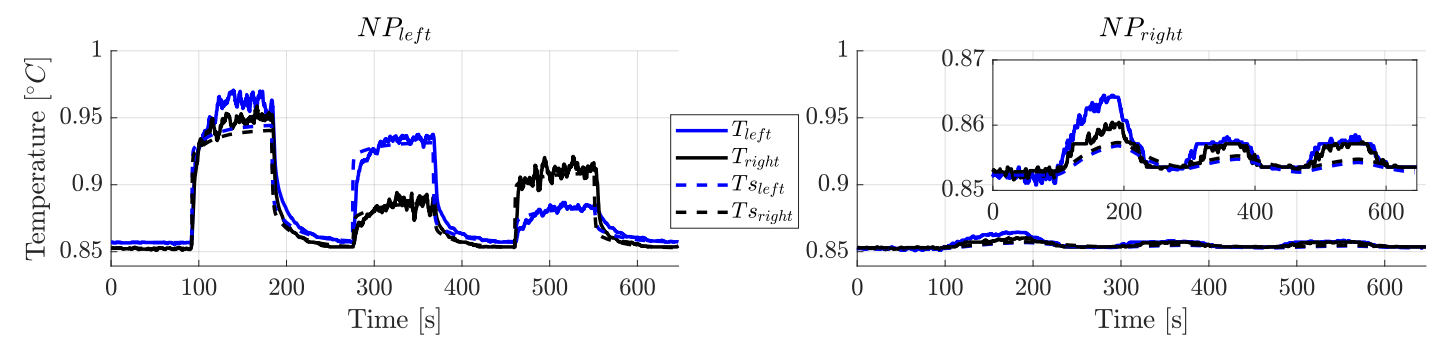

Fig. 6: Experimental validation of the model. $T_{\text {left }}, T_{\text {right }}$ are corresponding to the NTC thermistors. $T_{\text {Sleft }}, T_{\text {Sright }}$ are corresponding to the modeled temperature of the respective nodes. Although the nozzles in $N P_{\text {right }}$ are not used for jetting, the thermo-fluidic cross-talk causes their temperature to increase. Data points are scaled by a fixed constant.
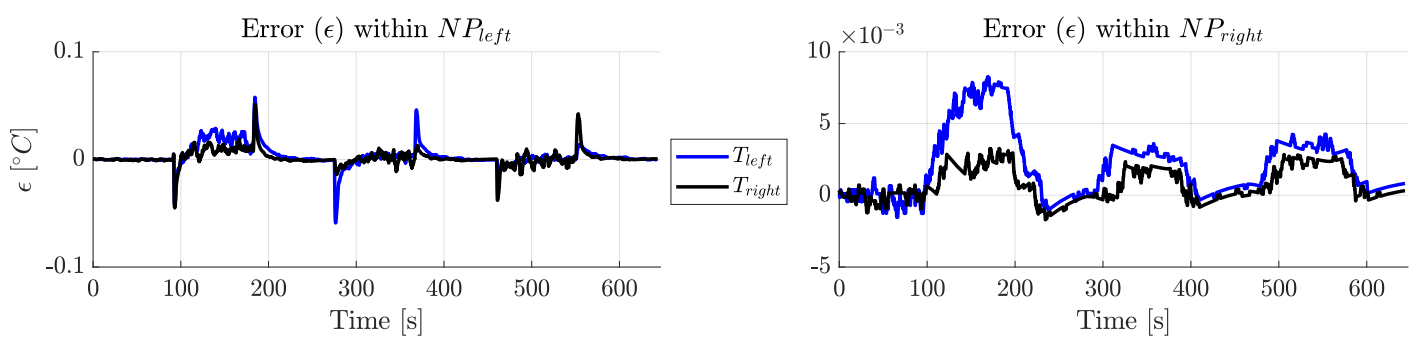

Fig. 7: Error between the developed model and experimental data on a normalized scale $\left(\epsilon:=T_{\text {left }}-T_{\text {Sleft }}, \epsilon:=T_{\text {right }}-\right.$ $T_{\text {Sright }}$ ) over time. Data points are scaled by a fixed constant.

temperatures of corresponding nodes simulated by the digital twin. The model-errors are plotted in figure 7

The developed model captures the thermo-fluidic behaviour of the printhead by indicating the offset in temperature among jetting and non-jetting nozzles. For example, as the nozzles in $N P_{\text {right }}$ are idle during the entire print-job, its temperature variation is significantly smaller. Nevertheless, there is still a small temperature variation for nozzles in $N P_{\text {right }}$ due to thermo-fluidic cross-talk among nozzles. After printing blank pages, while using every nozzle in $N P_{\text {left }}$, the temperature at $T_{\text {left }}$ ) and $T_{\text {right }}$ are close to each other. However, using only left-half of the nozzles or right-half of the nozzles in $N P_{\text {left }}$, among nozzles, there is a significant temperature difference over time (as depicted by large difference between $T_{\text {left }}$ and $T_{\text {right }}$ ). The role of the ink-return is also evident as the temperatures of the NP always go to a steady-state value while being idle.

\section{DEVELOPMENT AND CALIBRATION OF SOFT SENSOR}

For control, monitoring, and fault-diagnosis of the thermofluidic processes, it is necessary to acquire real-time information about the ink temperature. However, this must be achieved without incorporating any additional sensors. To this end, the piezoelectric elements that are located at every individual nozzle becomes useful. Apart from jetting ink droplets, the self-sensing capability of the piezoelectric actuator (c.f. [15]) is now calibrated to make a sensing device that provides ink temperature at every individual nozzle.

A piezoelectric element work in two operating modes. For jetting droplets of ink, the piezoelectric element is in actuation mode where a sequence of trapezoidal voltage pulses allows droplet formation and ejection [2], [16]. Once the pulse is applied, after a time delay $\left(t_{\text {delay }}\right)$, it is possible to connect the piezoelectric element with sensing circuitry and capture the residual pressure oscillations (this is also known as acousticsensing) [17]. Now, the piezoelectric element is in sensing mode. One can switch the operation of piezoelectric element by using an external trigger. A detailed description about the sensing-actuation mode of piezoelectric element, corresponding driving and triggering circuits and signal processing related aspects are explained in [4](pp. 29-30).

Signal measured from the acoustic sensing provides useful information about the dynamics inside a nozzle [2], [4], [18]. For instance, an experiment is performed where a particular nozzle is used to jet ink at two different temperatures, $T_{1}^{\circ} \mathrm{C}$ and $T_{2}^{\circ} \mathrm{C}$ with $T_{1}<T_{2}$. Corresponding acoustic sensing signals are measured and shown in figure 8 The difference in the measured signals indicates that the acoustic sensing signals can be used for estimating ink temperature.

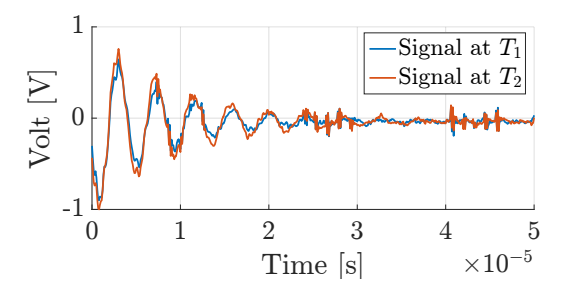

Fig. 8: Samples of measured acoustic sensing signal in a particular nozzle at two different ink temperatures $T_{1}, T_{2}$. Data points are scaled by a fixed constant. 


\section{A. Modeling Acoustic Sensing Signal}

In this paper, the acoustic sensing signal is used to estimate the ink temperature without employing any additional sensors. Let the acoustic sensing signal be discrete samples of a damped sinusoidal function:

$$
y(n)=\alpha e^{-\zeta n T_{s}} \sin \left(\omega n T_{s}+\phi\right)+\gamma ; \quad n \in \mathbb{N} .
$$

With a fixed sampling time $T_{s}, \alpha>0$ denotes the amplitude (Volt), $0<\omega<\pi$ the frequency (rad/sample), $-\pi<\phi \leq \pi$ the phase shift (rad), $\gamma$ the signal offset (Volt) and $\zeta \geq 0$ the damping factor.

\section{B. Determining Parameters $\alpha, \zeta, \omega, \gamma$ and $\phi$}

At first, the aim is to estimate the parameters $\alpha, \zeta, \omega, \gamma$ and $\phi$ such that 12 optimally approximates the measured acoustic sensing signals. To this end, let the acoustic sensing signal be the impulse response of a signal generating model as depicted in figure 9. As the output signal is modeled

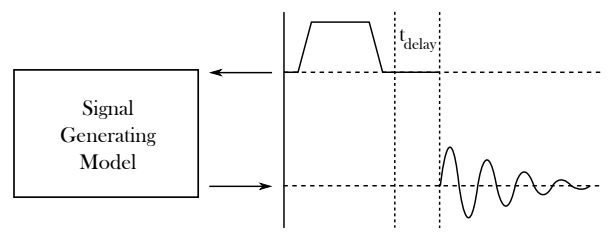

Fig. 9: Input-output relation for acoustic sensing of piezoelectric element

as damped sinusoidal function, the signal generating model belongs to a class of stable, second-order, single-input-singleoutput systems and has the following representation:

$$
\begin{aligned}
z(n+1) & =A_{s} z(n)+B_{s} w(n), \\
y(n) & =C_{s} z(n) .
\end{aligned}
$$

Here, for $n \in \mathbb{N} \cup\{0\}, z(n) \in \mathbb{R}^{2}$ is a vector with two internal states and $w(n) \in \mathbb{R}$ is applied impulse input. The output signal $y(n) \in \mathbb{R}$ is modeled as (12). A finite sequence of the output's samples are measured as $\left\{s_{n} \mid n \in \mathbb{N}_{[1, N]}\right\}$, where length of the data-set is $0<N<\infty$ and the sampling period is fixed as $T_{s}$.

Algorithm 1 presents a step-wise procedure to estimate $\alpha$, $\zeta, \omega, \gamma$ and $\phi$ using the measured data $\left\{s_{n} \mid n \in \mathbb{N}_{[1, N]}\right\}$.

\section{Calibration of The Relation between Acoustic Energy and Temperature}

Ink temperature is closely related to the estimated acoustic sensing signal. Let the acoustic energy be defined as the squared 2-norm of the acoustic sensing signal, $\|y(n)\|_{2}^{2}$. One expects lower acoustic energy in a lower temperature, and higher acoustic energy in a higher ink temperature. Now, the objective is to determine the relation between acoustic energy and temperature and use it to estimate ink temperature. This is achieved by performing three consecutive experiments.

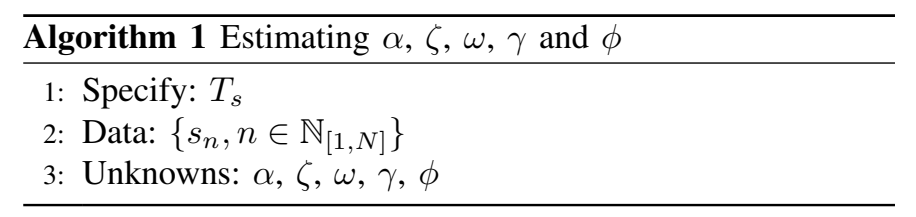

Step 1 - Finding poles of the signal generating model

4: Specify: $L \leftarrow\left\lfloor\frac{N}{3}\right\rfloor$

5: Construct Hankel matrix $H$ :

$$
H \leftarrow\left[\begin{array}{cccc}
s_{1} & \ldots & s_{L-1} & s_{L} \\
s_{2} & \ldots & s_{L} & s_{L+2} \\
\vdots & \ddots & \vdots & \vdots \\
s_{N-L} & \ldots & s_{N-2} & s_{N-1} \\
s_{N-L+1} & \ldots & s_{N-1} & s_{N}
\end{array}\right]
$$

6: Find optimal 2-rank approximation of $H$ by taking its singular value decomposition:

$$
H \approx U \Sigma V^{\mathrm{H}}, \quad \Sigma:=\operatorname{diag}\left(\sigma_{1}, \sigma_{2}\right), \quad \sigma_{1}, \sigma_{2}>0
$$

7: Define: $U^{1} \leftarrow U[1: N-L,:]$

8: Define: $U^{2} \leftarrow U[2: N-L+1,:]$

9: Determine: $\quad \tilde{A}_{s} \leftarrow \Sigma^{-\frac{1}{2}} U^{1^{\dagger}} U^{2} \Sigma^{\frac{1}{2}}$

10: for $k=1$ and 2 do

11: $\quad$ Solve eigen value $\rho_{k}$ and right eigen vector $v_{k}$ :

$$
\left(\tilde{A}_{s}-\rho_{k}\right) v_{k}=0
$$

\section{2: $\quad$ return $\rho_{k}$}

13: end for

Step $2-$ Determine $\omega$ and $\zeta$

14: for $k=1$ or 2 do

15: $\quad \omega \leftarrow \operatorname{Im}\left(\ln \rho_{k}\right)$

16: $\quad \zeta \leftarrow \operatorname{Re}\left(\ln \rho_{k}\right)$

7: end for

18: return $\omega, \zeta$

Step 3 - Determine $\alpha, \phi$, and $\gamma$

19: Define: $\mathbf{s} \leftarrow \operatorname{col}\left(s_{1}, \cdots, s_{N}\right)$

20: for $n \in \mathbb{N}_{[1, N]}$ do

21: $\quad \boldsymbol{\Gamma}[n, 1] \leftarrow\left(e^{j(\omega+j \zeta) n T_{s}}+e^{-j(\omega-j \zeta) n T_{s}}\right)$

22: $\quad \boldsymbol{\Gamma}[n, 2] \leftarrow j\left(e^{j(\omega+j \zeta) n T_{s}}-e^{-j(\omega-j \zeta) n T_{s}}\right)$

23: $\quad \boldsymbol{\Gamma}[n, 3] \leftarrow 1$

24: end for

25: Solve $\hat{\mathbf{x}}=\arg \min _{\mathbf{x}}\|\boldsymbol{\Gamma} \mathbf{x}-\mathbf{s}\|_{2}$ :

$$
\hat{\mathbf{x}} \leftarrow\left(\boldsymbol{\Gamma}^{\mathrm{H}} \boldsymbol{\Gamma}\right)^{-1} \boldsymbol{\Gamma}^{\mathrm{H}} \mathbf{S}
$$

$$
\begin{aligned}
& \text { 26: } \alpha \leftarrow 2 \sqrt{\operatorname{Re}(\hat{\mathbf{x}}[1]+j \hat{\mathbf{x}}[2])^{2}+\operatorname{Im}(\hat{\mathbf{x}}[1]+j \hat{\mathbf{x}}[2])^{2}} \\
& \text { 27: } \phi \leftarrow \operatorname{Im}(\ln (\hat{\mathbf{x}}[1]+j \hat{\mathbf{x}}[2])) . \\
& \text { 28: } \gamma \leftarrow \hat{\mathbf{x}}[3] \\
& \text { 29: } \operatorname{return} \alpha, \phi, \gamma
\end{aligned}
$$

1) Experiment 1: At first, to verify the relation between ink temperature and acoustic energy, an experiment is performed. In this experiment, three operating points are chosen for the ink temperature. At a fixed operating point of temperature, 
the ink is jetted using the actuation mode of the piezoelectric element. After that, it is switched to sensing mode 20 times consecutively, and each time the acoustic sensing signal is measured. Every individual signal contains 100 samples of measured data. Using the measured data, the acoustic sensing signal is reconstructed using Algorithm 1 and its energy is computed. Based on this experiment, the energy-temperature

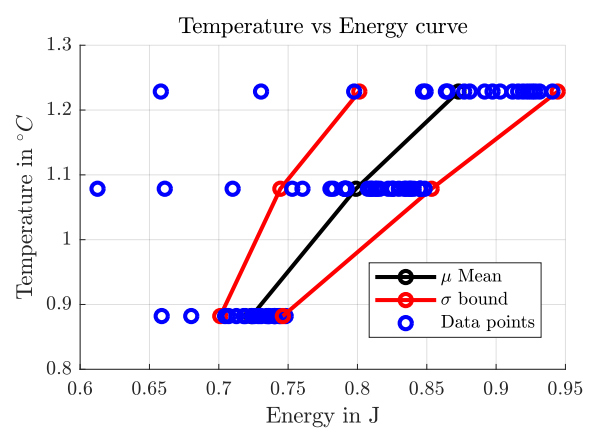

Fig. 10: Mean and variance of energy-temperature curve at three operating points of ink temperatures. Data points are scaled by a fixed constant.

curve is shown in figure 10. Despite the expected trend in change of acoustic energy with temperature, the variation of energy estimate is significantly large. Therefore, estimating ink temperature using energy-temperature curve is still unreliable.

2) Experiment 2: The evaporation of liquid changes its viscosity and temperature [13], hence, the acoustic energy. In order to present the consequence of evaporation, an experiment, similar to Experiment 1, is performed where four operating points of temperature are chosen. Ink is jetted at these operating temperature by using 2000 consecutive jetting pulses. The last jetting pulse is, thereafter, followed by switching the piezoelectric element in sensing mode and measuring a sequence of acoustic sensing signals. Here, the aim is to investigate the evolution of acoustic energy over time. In figure 11, the estimated acoustic energy of the acoustic

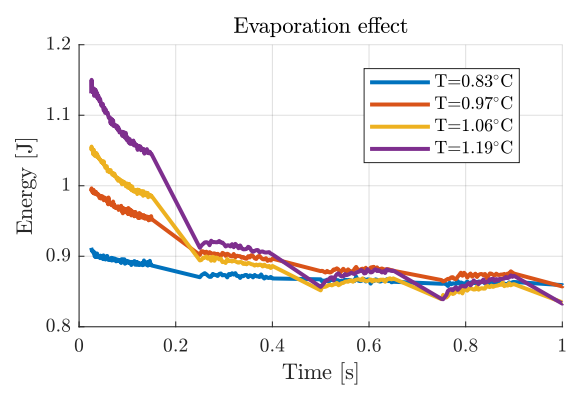

Fig. 11: Effect of evaporation on the estimated acoustic energy over time. Data points are scaled by a fixed constant.

signal is shown over one second. Over time, the effect of evaporation results in an initial decay of energy and the decay rate is higher if temperature of the ink increases. After 0.5 seconds, one observes a periodic fluctuation in the energy which can be explained by change in evaporation enthalpy due to the mixing between inlet ink and the ink that is already present in the nozzle. This experiment implies that, due to evaporation, estimating the relation between temperature and acoustic energy depends on the time sequence at which the acoustic sensing signals are processed to determine the energy. In other words, larger the difference between time of jetting pulses and the time sequence of the measured signal, lower the acoustic energy leading to a poor signal to noise ratio.

3) Experiment 3: To circumvent the effect of evaporation from the energy-temperature relation, only those acoustic sensing signals should be considered that are measured immediately after the jetting pulse. To this end, another experiment is performed where the ink temperature is varied over four operating points. This time, instead of measuring multiple acoustic sensing signals one after another, jetting of ink is directly followed by the sensing of acoustic sensing signal. This sequence of consecutive jetting and sensing is repeated 20 times. Using the measured data, the acoustic energy is estimated. Figure 12 shows that the adapted approach of

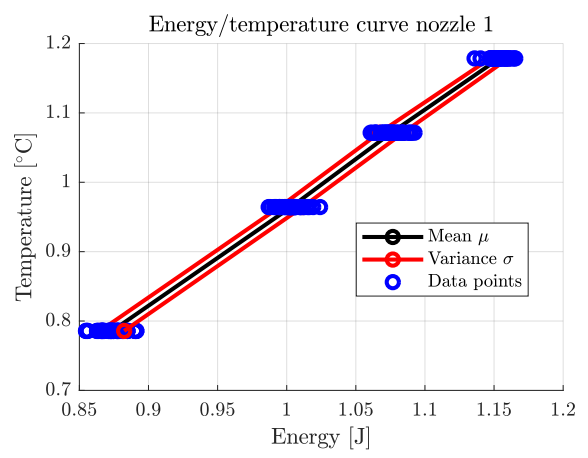

Fig. 12: Relation between the ink temperature of a nozzle and estimated acoustic energy. Data points are scaled by a fixed constant. The variance of energy-temperature relation significantly improves by adapting the jetting-sensing sequence.

consecutive jetting-sensing significantly improves the variance in the estimate of energy-temperature relation.

\section{Estimation of Ink Temperature and Ink Viscosity Using Energy-Temperature Curve}

In the previous exposition, using consecutive sequence of jetting and sensing, the measured acoustic sensing signals are used to estimate the acoustic energy and calibrate the corresponding energy-temperature curve for various operating points of ink temperature. Now, using linear regression, one can determine a parametric linear model relating acoustic energy and ink temperature. Let this model of energytemperature relation be given by:

$$
T=m \phi+c
$$

where $T$ is the ink temperature and $\phi$ is the acoustic energy defined as the squared 2-norm of the acoustic sensing signal, $\|y(n)\|_{2}^{2}$. The unknowns are $m, c \in \mathbb{R}$ that can be obtained based on the data of Experiment 3. According to (14), once the energy-temperature curve is obtained, it can be used for estimating ink temperature with new measurements. Every time a liquid droplet is jetted, thereafter, it can be followed by measuring acoustic sensing signal. Using Algorithm 1, the 
acoustic energy is modeled as (12) and its acoustic energy is determined. Corresponding ink temperature is determined by the energy-temperature curve in (14). In figure 13, energy-

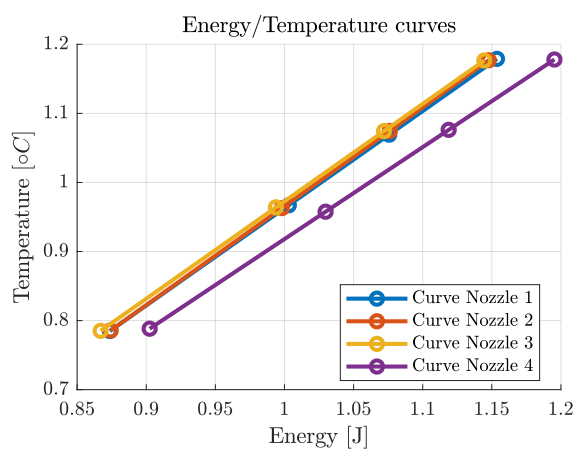

Fig. 13: Estimation of energy-temperature curve for four nozzles. Data points are scaled by a fixed constant.

temperature curves are estimated for four different nozzles in the printhead.

One can also use the temperature estimate to infer the change in viscosity of the ink. Specifically, using the viscosity-temperature curve for a particular ink material (c.f. the open source data-sheet in the link, https://wiki.anton-paar.com/en/ink/), one is able to estimate the variation of viscosity with respect to the signal energy. In figure 14, energy-viscosity curves are estimated for four nozzles.

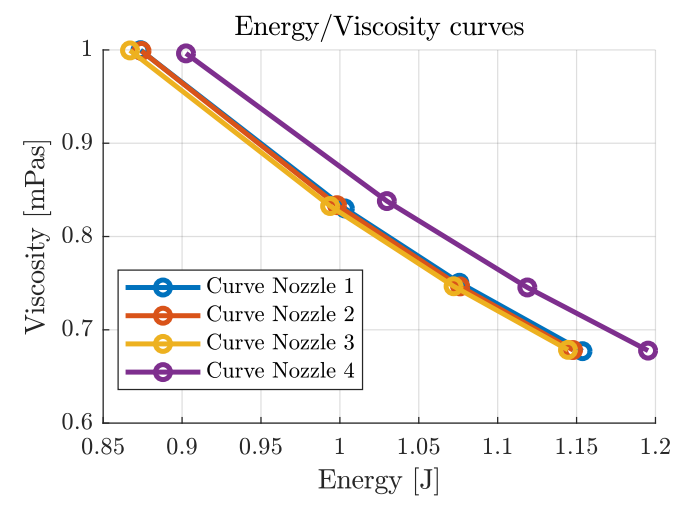

Fig. 14: Estimation of viscosity for four nozzles on a normalized scale.

\section{CONTROLLING INK TEMPERATURE IN NOZZLES: A PROOF OF PRINCIPLE}

The performance limiting aspect of thermo-fluidic processes is the fluctuation in ink temperature among adjacent nozzles in a printhead as well among adjacent printheads. To maintain temperature consistency, in this section, a controller is synthesized that does not require incorporating any additional sensor or actuator. The only resources the controller uses are a) the bitmap as a prior knowledge about the flow pattern in the printhead, b) the model to anticipate and predict the evolution of thermo-fluidic behaviour in the printhead, and c) the softsensor as a feedback information on temperature and viscosity at every nozzle.

\section{A. Concept of In Situ Sensing-Actuation}

Every individual nozzle is equipped with a piezoelectric element for jetting ink. By applying voltage, its resistive property allows the piezoelectric element to dissipate heat. In this controller design problem, the resistive property of a jetting actuator is used as a source of heating. Thus, the piezoelectric element makes every individual nozzle equipped with a local, independent, in situ self-sensing actuator.

However, the piezoelectric elements can only be used as heaters for control input when there is no need for jetting liquid ink. To implement the in situ sensing-actuation scheme, one requires to classify the nozzles based on their modes of operations. At time $t \in \mathbb{T}$, the nozzles are allocated in four categories defined by the following set-valued maps:

1) Jetting nozzles, $\mathbb{S}_{p}(t)$ : Set of $n_{p}$ nozzles that are used for jetting liquid droplets.

2) Heating nozzles, $\mathbb{S}_{h}(t)$ : Set of $n_{h}$ nozzles that are nonjetting and are used as heater.

3) Sensing nozzles, $\mathbb{S}_{s}(t)$ : Set of $n_{s}$ nozzles whose temperature or viscosity are sensed.

4) Idle nozzles, $\mathbb{S}_{u}(t)$ : Set of $n_{u}$ nozzles that are neither in any of the above modes.

Given the set of all nozzles $\mathbb{S}$, the above set-valued maps are pre-determined by the bit-map depending on the entire printjob. With varying bit-map, the cardinality of individual set also changes. Additionally, one must ensure $\mathbb{S}_{h} \subseteq \mathbb{S} \backslash \mathbb{S}_{p}$ for all time $t \in \mathbb{T}$.

\section{B. Performance Specifications}

The controller must satisfy the following performance specification:

1) Irrespective of the print-job, the differences in temperature among adjacent nozzles must be as small as possible.

2) The usage of thermal actuation must be limited to sustain operational lifetime of piezoelectric elements.

3) Cooling of the liquid ink is not possible using the thermal actuation.

4) Thermal actuation of piezoelectric material should be non-jetting, i.e. it should not form new droplets of liquid.

Intuitively, using the in situ sensing-actuation scheme, the controller is expected to actuate few heating nozzles (from the set $\mathbb{S}_{h}$ ) adjacent to the jetting ones to limit the temperature difference among nozzles while satisfying the performance specifications. At the same time, the nozzles' temperature can be monitored using the implemented soft sensor at the sensing nozzles (from the set $\mathbb{S}_{s}$ ).

\section{Generating Voltage Pulse for Heating Actuators}

To apply the required amount of thermal power as control inputs, one needs to design voltage pulses that are to be applied on the piezoelectric elements in heating nozzles. Such voltage pulse must not cause ejection of droplets while satisfying the power requirement. The principle behind generating non-jetting voltage pulse is to modulate the amplitude and frequency of trapezoidal pulses (c.f. [19]). Recently, in [20], a mechanism is developed so as to generate specific thermal 
energy while not interfering with frequency region of jetting. Here, using band-pass modulation, the width and the height of the trapezoidal pulses are tuned.

\section{Model-Based Prediction of Ink Temperature in Nozzles}

In this paper, there are four equivalent ways to represent the model of thermo-fluidic processes and one has the freedom to use any one of them for predicting the future evolution of ink temperature in nozzles. Here, the thermo-fluidic behaviour is considered to be represented according to (9). In discrete time, i.e. for $t \in\left\{k t_{d} \mid k \in \mathbb{N} \cup\{0\}\right\}$, the behaviour is governed by the following equations:

$$
\left[\begin{array}{c}
\left(Q_{t}(x)\right)(t) \\
y(t)
\end{array}\right]=\left[\begin{array}{ll}
\tilde{A}(t) & \tilde{B}(t) \\
\tilde{C}(t) & \tilde{D}(t)
\end{array}\right]\left[\begin{array}{l}
x(t) \\
u(t)
\end{array}\right]+\left[\begin{array}{c}
G(t)+W(t) d(t) \\
J(t)
\end{array}\right]
$$

Here, $\left(Q_{t}(x)\right)(t):=x\left(t+t_{d}\right), t \in\left\{k t_{d} \mid k \in \mathbb{N} \cup\{0\}\right\}$. If there are $n_{\text {nozzle }}$ number of nozzles, $u(t), y(t) \in \mathbb{R}^{n_{\text {nozzle }}}$.

During jetting, the actuation of jetting piezoelectric actuators causes heat dissipation [19]. Given the amplitude and frequency of the voltage pulses, one can estimate the thermal power (in Watt) generated in jetting nozzles over time and they are modeled as known disturbance $d(t) \in \mathbb{R}^{n_{\text {nozzle }}}$.

However, to implement the in situ sensing-actuation scheme, the bit-map allocates the nozzles according four modes of operation. To map the total number of nozzles to its allocated modes of operation, binary matrices $S^{p}(t) \in \mathbb{R}^{n_{\text {nozle }} \times n_{p}(t)}$, $S^{h}(t) \in \mathbb{R}^{n_{\text {nozzle }} \times n_{h}(t)}$, and $S^{s}(t) \in \mathbb{R}^{n_{s}(t) \times n_{\text {nozzle }}}$ are introduced. Their $(i, j)$-th entries are:

$$
\begin{aligned}
& S^{p}(i, j)(t)= \begin{cases}1, & i \in \mathbb{S}_{p}(t), \quad j \in \mathbb{N}_{\left[1, n_{p}(t)\right]}, \\
0, & \text { otherwise. }\end{cases} \\
& S^{h}(i, j)(t)= \begin{cases}1, & i \in \mathbb{S}_{h}(t), \quad j \in \mathbb{N}_{\left[1, n_{h}(t)\right]}, \\
0, & \text { otherwise }\end{cases} \\
& S^{s}(i, j)(t)= \begin{cases}1, & i \in \mathbb{N}_{\left[1, n_{s}(t)\right]}, \quad j \in \mathbb{S}_{s}(t), \\
0, & \text { otherwise. }\end{cases}
\end{aligned}
$$

As a result, 15] is modified as

$$
\begin{aligned}
{\left[\begin{array}{c}
\left(Q_{t}(x)\right)(t) \\
y^{s}(t)
\end{array}\right]=} & {\left[\begin{array}{cc}
\tilde{A}(t) & \tilde{B}(t) S^{h}(t) \\
S^{s}(t) \tilde{C}(t) & S^{s}(t) \tilde{D}(t) S^{h}(t)
\end{array}\right]\left[\begin{array}{c}
x(t) \\
u^{h}(t)
\end{array}\right] } \\
& +\left[\begin{array}{c}
G(t)+W(t) S^{p}(t) d^{p}(t) \\
J(t)
\end{array}\right]
\end{aligned}
$$

Here, at time $t \in \mathbb{T}$, the control inputs $u^{h}(t) \in \mathbb{R}^{n_{h}(t)}$ are applied by the set of heating nozzles (in terms of thermal power, Watt). The disturbance due to jetting $d^{p}(t) \in \mathbb{R}^{n_{p}(t)}$ are applied by the set of jetting nozzles (in terms of thermal power, Watt). The sensed outputs $y^{s}(t) \in \mathbb{R}^{n_{s}(t)}$ are sensed ink temperature by the set of sensing nozzles (in terms of temperature, ${ }^{\circ} \mathrm{C}$ ).

\section{E. Formulating Operational Constraints}

Let $u_{\max } \in \mathbb{R}^{n_{\text {nozzle }}}$ be the maximum admissible thermal power of all piezoelectric elements. Here, $u_{\max }$ is determined such that it takes into the degradation of piezoelectric elements and not ejecting new droplet of liquid ink. On the other end, cooling is not allowed. As a result, at every time instant $t \in \mathbb{T}$, heating piezoelectric elements must satisfy the constraint

$$
0 \leq S^{h}(t) u^{h}(t) \leq u_{\max }
$$

Having bounded actuation and bounded thermal disturbance during jetting, no additional constraints on the state variable $x(t)$ has been enforced.

\section{F. Specifying Control Criterion}

The purpose of the controller is to control the temperature difference among nozzles. They are defined as

$$
z(t)=H x(t),
$$

where, $z$ denotes the vector of all temperature differences among individual adjacent nozzles. In particular, $H \in \mathbb{R}^{n_{x} \times n_{x}}$ is a sparse matrix with $H_{i, i}=1$ and $H_{i, j}=-1$ whenever $i^{\text {th }}$ nozzle is adjacent to the $j^{\text {th }}$ nozzle.

At every time instant $t=k t_{d}$ with $k \in \mathbb{N} \cup\{0\}$, the requirement is to control the ink temperature gradient among nozzles over a finite horizon of future time instants $t \in \mathbb{T}_{N}^{k}$ where $\mathbb{T}_{N}^{k}:=\left\{k t_{d} \mid k \in \mathbb{N}_{[k, N+k]}\right\}$ for $N>0$. To this end, a reference tracking problem is formulated to minimize the following cost functional:

$$
J_{k}:=\sum_{t \in \mathbb{T}_{N}^{k}}\left\|x(t)-x^{r}\right\|_{Q_{k}(t)}^{2}+\sum_{t \in \mathbb{T}_{N-1, k}}\left\|u^{h}(t)-u^{r}(t)\right\|_{R_{k}(t)}^{2} .
$$

Here, the reference trajectories $\left(x^{r}, u^{r}(t)\right)$ are pre-determined from a steady state model that equates the temperature difference of nozzles to zero. In other words, for $t \in \mathbb{T}_{N}^{k},\left(x^{r}, u^{r}(t)\right)$ are the solution of the following linear equations:

$$
\begin{aligned}
x^{r} & =\tilde{A}(t) x^{r}+\tilde{B}(t) S^{h}(t) u^{r}(t)+G(t)+W(t) S^{p}(t) d^{p}(t), \\
0 & =H x^{r},
\end{aligned}
$$

Moreover, for all $t \in \mathbb{T}_{N}^{k}$, one must pre-define the weights $Q_{k}(t) \in \mathbb{R}^{n_{x} \times n_{x}}, R_{k}(t) \in \mathbb{R}^{n_{h}(t) \times n_{h}(t)}$ to penalize the deviation of states and inputs from their respective references.

\section{G. Formulating Model Predictive Control Scheme}

A digital controller is required that minimizes 19 ) by using future prediction on the states in 16 while satisfying the constraints in 177. To this end, a reference-tracking model predictive control (MPC) scheme is presented (c.f. [21]).

At every time instant $t=k t_{d}$ with $k \in \mathbb{N} \cup\{0\}$, for convenience, let any function $f(t)$ be defined as $f_{0 \mid k}:=f\left(k t_{d}\right)$. From its initial value $f_{0 \mid k}$, over a finite horizon of future time instants $t \in \mathbb{T}_{N}^{k}$ with $N>0$, the value of $f(t)$ at instant $k+i$ is denoted by $f_{i \mid k}:=f\left((k+i) t_{d}\right)$. Starting from the time step $k t_{d}$, the $N$-horizon MPC scheme amounts to minimizing the following cost functional

$$
J\left(k, \bar{x}_{k}, \bar{u}_{k}^{h}\right):=\left\|x_{N \mid k}-x^{r}\right\|_{Q_{k}}^{2}+
$$




$$
\sum_{i=0}^{N-1}\left(\left\|x_{i \mid k}-x^{r}\right\|_{Q}^{2}+\left\|u_{i \mid k}^{h}-u_{i \mid k}^{r}\right\|_{R_{i \mid k}}^{2}\right),
$$

subject to the following constraints:

$$
\begin{aligned}
x_{i+1 \mid k} & =\tilde{A}_{i \mid k} x_{i \mid k}+\tilde{B}_{i \mid k} S_{i \mid k}^{h} u_{i \mid k}^{h}+\tilde{G}_{i \mid k}, i \in \mathbb{N}_{[0, N-1]}, \\
E_{i \mid k} u_{i \mid k}^{h} & \leq b, \quad i \in \mathbb{N}_{[0, N-1]}, \\
F_{N \mid k} x_{N \mid k} & \leq b_{N \mid k}, \\
x_{0 \mid k} & =x\left(k t_{d}\right) .
\end{aligned}
$$

Here, for $i \in \mathbb{N}_{[0, N-1]}$,

$$
\begin{aligned}
\tilde{G}_{i \mid k} & =G_{i \mid k}+W_{i \mid k} S_{i \mid k}^{p} d_{i \mid k}^{p}, \\
E_{i \mid k} & =\operatorname{col}\left(-S_{i \mid k}^{h}, S_{i \mid k}^{h}\right), \\
b & =\operatorname{col}\left(0, u_{\max }\right) .
\end{aligned}
$$

For all $t \in \mathbb{T}_{N}^{k}$, the reference trajectories $\left(x^{r}, u^{r}\right)$ are determined by solving 20 . The weights $Q \in \mathbb{R}^{n_{x} \times n_{x}}, R_{i \mid k} \in$ $\mathbb{R}^{n_{h}(t) \times n_{h}(t)}$ are user-defined. The terminal penalty $Q_{k} \in$ $\mathbb{R}^{n_{x} \times n_{x}}$ in the cost 21] and $F_{N \mid k}, b_{N \mid k}$ in the terminal constraint 22c are specifically chosen to ensure stability of the closed-loop system [22].

The above constrained optimization is solved for $N$ horizons over the sequence of predicted states and future inputs that are denoted as $\bar{x}_{k}:=\left\{x_{1 \mid k}, \cdots, x_{N \mid k}\right\}$ and $\bar{u}_{k}:=$ $\left\{u_{0 \mid k}, \cdots, u_{N-1 \mid k}\right\}$ respectively. If the minimizer of the optimal control problem (21)-(22) is denoted by $\bar{u}_{k}^{h *}:=$ $\left\{u_{0 \mid k}^{h *}, \cdots, u_{N-1 \mid k}^{h *}\right\}$, then its first entry $u_{0 \mid k}^{h *}$ is implemented as control input at time step $t=k t_{d}$. Subsequently, on a receding horizon, the optimal control problem is solved again at step $(k+1) t_{d}$ using $x_{0 \mid k+1}=x\left((k+1) t_{d}\right)$ as its initial state. In a receding horizon fashion, this procedure is continued iteratively over all time $t \in\left\{k t_{d} \mid k \in \mathbb{N} \cup\{0\}\right\}[23$.

Theorem VI.1. Given the matrices $R_{i \mid k} \in \mathbb{S}_{\succ 0}^{n_{h}(t)}, Q \in \mathbb{S}_{\succ 0}^{n_{x}}$ for all time $t \in \mathbb{T}_{N}^{k}$, let the $N$-horizon MPC has a unique solution $\left\{u_{0 \mid k}^{h *}, \cdots, u_{N-1 \mid k}^{h *}\right\}$ that minimizes (21) subject to (22) under the following conditions.

1) If there exist feasible matrices $X_{k} \in \mathbb{S}_{\succ 0}^{n_{x}}$ and $Y_{k} \in$ $\mathbb{R}^{n_{h}(t) \times n_{x}}$ that satisfy the following linear matrix inequality (LMI)

$$
\left(\begin{array}{cccc}
-X_{k} & 0 & \tilde{A}_{N \mid k} X_{k}+\tilde{B}_{N \mid k} Y_{k} & 0 \\
0 & -R_{N \mid k}^{-1} & Y_{k} & 0 \\
\left(\tilde{A}_{N \mid k} X_{k}+\tilde{B}_{N \mid k} Y_{k}\right)^{\top} & Y_{k}^{\top} & -X_{k} & X_{k} \\
0 & 0 & X_{k} & -Q^{-1}
\end{array}\right) \preccurlyeq 0,
$$

then the matrix $Q_{k}$ must satisfy $Q_{k}=X_{k}^{-1}$ in (21).

2) The terminal constraint (22c) must satisfy

$$
E_{N \mid k} K_{k} x_{N \mid k} \leq b-u_{N \mid k}^{r}+E_{N \mid k} K_{k} x^{r}
$$

where, $K_{k}=Y_{k} X_{k}^{-1}$ is obtained using the feasible solution of 23. Moreover, $E_{N \mid k}=\operatorname{col}\left(-S_{N \mid k}^{h}, S_{N \mid k}^{h}\right)$, $b=\operatorname{col}\left(0, u_{\max }\right)$.

Then, once the control input $u^{h}\left(k t_{d}\right)=u_{0 \mid k}^{h *}$ is applied to (16), there exists a $\delta>0$ such that $\lim \left\|x\left(k t_{d}\right)-x^{r}\right\|_{2}=0$ for all initial condition $\left\|x(0)-x^{r}\right\|_{2}<\delta$.
One may substitute the equality constraint $22 \mathrm{a}$ in (21) to eliminate the variables $\left\{x_{0 \mid k}, \cdots, x_{N \mid k}\right\}$. This results in the following dense linearly constrained quadratic programming (LCQP) problem:

Optimization Problem 1. (Dense LCQP)

$$
\begin{aligned}
& \quad \arg \min _{U_{k}} \frac{1}{2} U_{k}^{\top} G_{k} U_{k}+U_{k}^{\top} W_{k} \\
& \text { subject to } L_{k} U_{k} \leq V_{k},
\end{aligned}
$$

where,

$$
\begin{aligned}
G_{k} & =2\left(\Gamma_{k}^{\top} \Omega_{k} \Gamma_{k}+\Psi_{k}\right), \\
W_{k} & =\left(2 \Gamma_{k}^{\top} \Omega_{k} \Phi_{k}\right) x_{0 \mid k}+2 \Gamma_{k}^{\top} \Omega_{k} \Xi_{k}-\Lambda_{k}, \\
\Lambda_{k} & =-2 \Gamma_{k}^{\top} \Omega_{k} X_{k}^{r}-2 \Psi_{k} U_{k}^{r}, \\
L_{k} & =\bar{M} \Gamma_{k}+\bar{E}_{k}, \\
V_{k} & =\bar{b}-\bar{M} \Phi_{k} x_{0 \mid k}-\bar{M} \Xi_{k}, \\
x_{0 \mid k} & =x(k),
\end{aligned}
$$

with

$$
\begin{aligned}
& \Phi_{k}=\left[\begin{array}{c}
\tilde{A}_{0 \mid k} \\
\tilde{A}_{1 \mid k} \tilde{A}_{0 \mid k} \\
\vdots \\
\tilde{A}_{N-1 \mid k} \cdots \tilde{A}_{0 \mid k}
\end{array}\right], \bar{M}=\left[\begin{array}{ccc}
0 & \cdots & 0 \\
0 & \cdots & 0 \\
\vdots & \ddots & \vdots \\
0 & \cdots & E_{N \mid k} K_{k}
\end{array}\right] \\
& \bar{E}_{k}=\left[\begin{array}{ccc}
E_{0 \mid k} & \cdots & 0 \\
\vdots & \ddots & \vdots \\
0 & \cdots & E_{N-1 \mid k} \\
0 & \cdots & 0
\end{array}\right], \bar{b}=\left[\begin{array}{c}
b \\
b \\
\vdots \\
b-u_{N \mid k}^{r}+E_{N \mid k} K_{k} x^{r}
\end{array}\right] \text {, } \\
& \Gamma_{k}=\left[\begin{array}{cccc}
\tilde{B}_{0 \mid k} & 0 & \cdots & 0 \\
\tilde{A}_{1 \mid k} B_{0 \mid k} & \tilde{B}_{1 \mid k} & \cdots & 0 \\
\vdots & \vdots & \ddots & \vdots \\
\tilde{A}_{N-1 \mid k} \cdots \tilde{A}_{1 \mid k} \tilde{B}_{0 \mid k} & \tilde{A}_{N-2} \cdots \tilde{A}_{2 \mid k} \tilde{B}_{1 \mid k} & \cdots & \tilde{B}_{N-1 \mid k}
\end{array}\right] \\
& \Xi_{k}=\left[\begin{array}{cccc}
\tilde{G}_{0 \mid k} & 0 & \cdots & 0 \\
\tilde{A}_{1 \mid k} \tilde{G}_{0 \mid k} & \tilde{G}_{1 \mid k} & \cdots & 0 \\
\vdots & \vdots & \ddots & \vdots \\
\tilde{A}_{N-1 \mid k} \cdot \tilde{A}_{1 \mid k} \tilde{G}_{0 \mid k} & \tilde{A}_{N-2 \mid k} \cdot \tilde{A}_{2 \mid k} \tilde{G}_{1 \mid k} & \cdots & \tilde{G}_{N-1 \mid k}
\end{array}\right]\left[\begin{array}{c}
1 \\
1 \\
\vdots \\
1
\end{array}\right] .
\end{aligned}
$$

Here, the decision variables are the to-be-applied control input $U_{k}=\operatorname{col}\left(u_{0 \mid k}, \cdots, u_{N-1 \mid k}\right)$. Moreover, $X_{k}^{r}=$ $\operatorname{col}\left(x^{r}, \cdots, x^{r}\right)$ and $U_{k}^{r}=\operatorname{col}\left(u_{0 \mid k}^{r}, \cdots, u_{N-1 \mid k}^{r}\right)$ are the reference values for states and inputs respectively. Moreover, $\Psi_{k}:=\operatorname{diag}\left(R_{0 \mid k}, \cdots, R_{N-1 \mid k}\right)$ and $\Omega_{k}:=$ $\operatorname{diag}\left(Q, \cdots, Q, Q_{k}\right)$.

\section{H. Implementation and Illustration of MPC Scheme}

As a proof of principle, the presented MPC scheme is implemented to minimize the difference in ink temperature among nozzles. To this end, the following specifications are considered:

1) Printhead Configuration: The printhead chosen for implementing MPC has the architecture shown in figure 1a. There are 48 nozzles that are equally divided over two-side of the $\mathrm{NP}$, and at each side, 24 nozzles are equally distributed over two rows. 


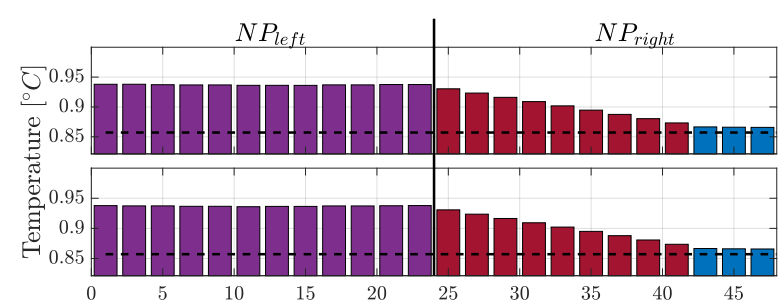

(a) Controlled Scenario 1: DM0 is fully used for jetting.

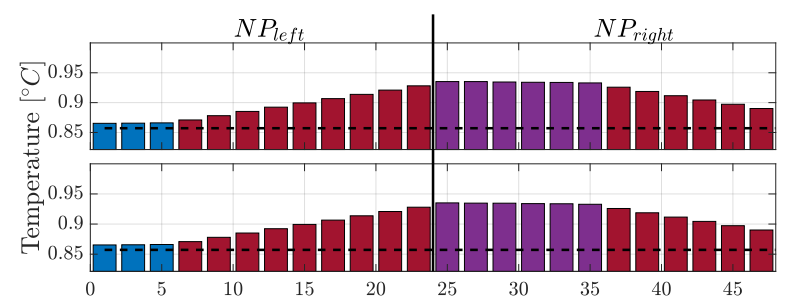

(a) Controlled Scenario 2: Left half of DM1 is used for jetting.

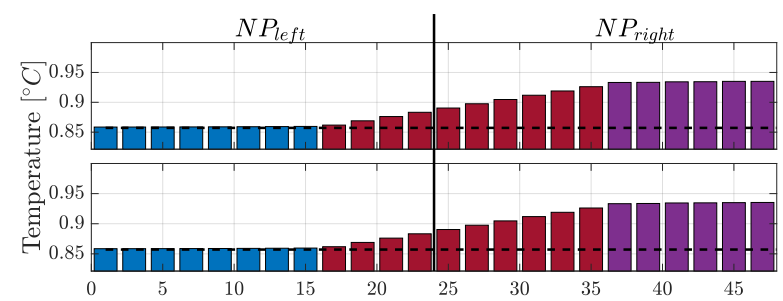

(a) Controlled Scenario 3: Right half of DM1 is used for jetting.

Fig. 17: Ink temperature at each nozzle for three scenarios in controlled and uncontrolled case. denotes nozzles that are jetting. denotes the adjacent nozzles that are used as thermal actuators. denotes the nozzles that are not used for heating. Data points are scaled by a fixed constant.

2) Printjob Specification: Generation of a test bit-map, the following scenarios are considered:

- Scenario 1: All 24 nozzles that located in left-side of NP (denoted as $N P_{l e f t}$ ) are used as jetting nozzle.

- Scenario 2: 12 nozzles in right-side of NP (denoted as $N P_{\text {right }}$ ) that are located closest to the $N P_{\text {left }}$ are used as jetting nozzle.

- Scenario 3: 12 nozzles in right-side of NP (denoted as $\left.N P_{\text {right }}\right)$ that are located farthest from the $N P_{\text {left }}$ are used as jetting nozzle.

3) Setting Up the Digital Twin of the Model: Based on the above scenarios, the ink flow parameters are assigned to every individual node. At the same time, the thermal power dissipated by individual jetting nozzle are used as known disturbance.

The model of every individual nodes is considered in discrete time by choosing the $\mathbb{T}=\left\{k t_{d} \mid k \in \mathbb{N} \cup\{0\}\right\}$. Euler's approach is used for time discretization due to its sparse and structure preserving implementation ( [14], page $4)$. For numerical stability, the sampling period $t_{d}$ is chosen to be 0.01 seconds.

Now, the thermo-fluidic model is defined as a graph following the definitions (D.1)-(D.3). The equivalent representation (9) is determined by eliminating the interconnection and print-

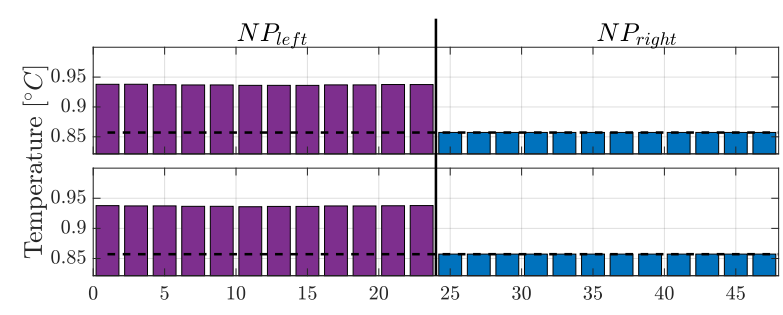

(b) Uncontrolled Scenario 1: DM0 is fully used for jetting.

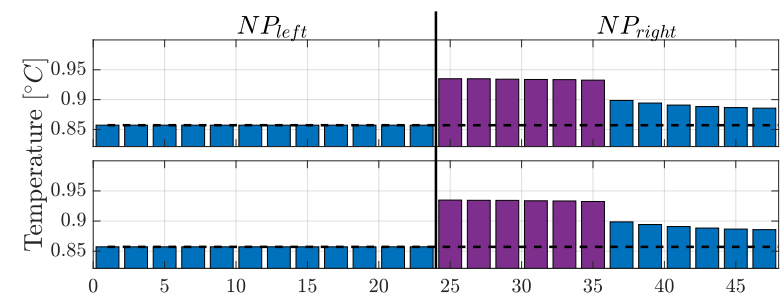

(b) Uncontrolled Scenario 2: Left half of DM1 is used for jetting.

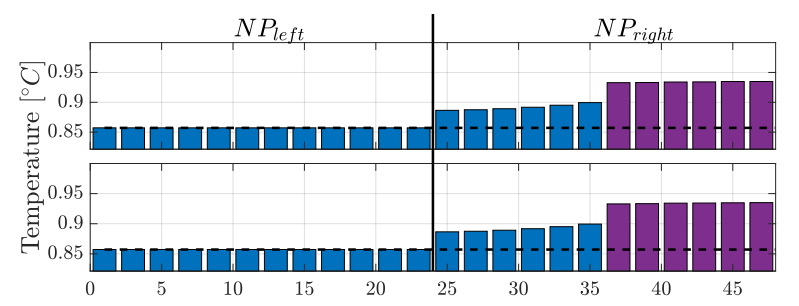

(b) Uncontrolled Scenario 3: Right half of DM1 is used for jetting. 


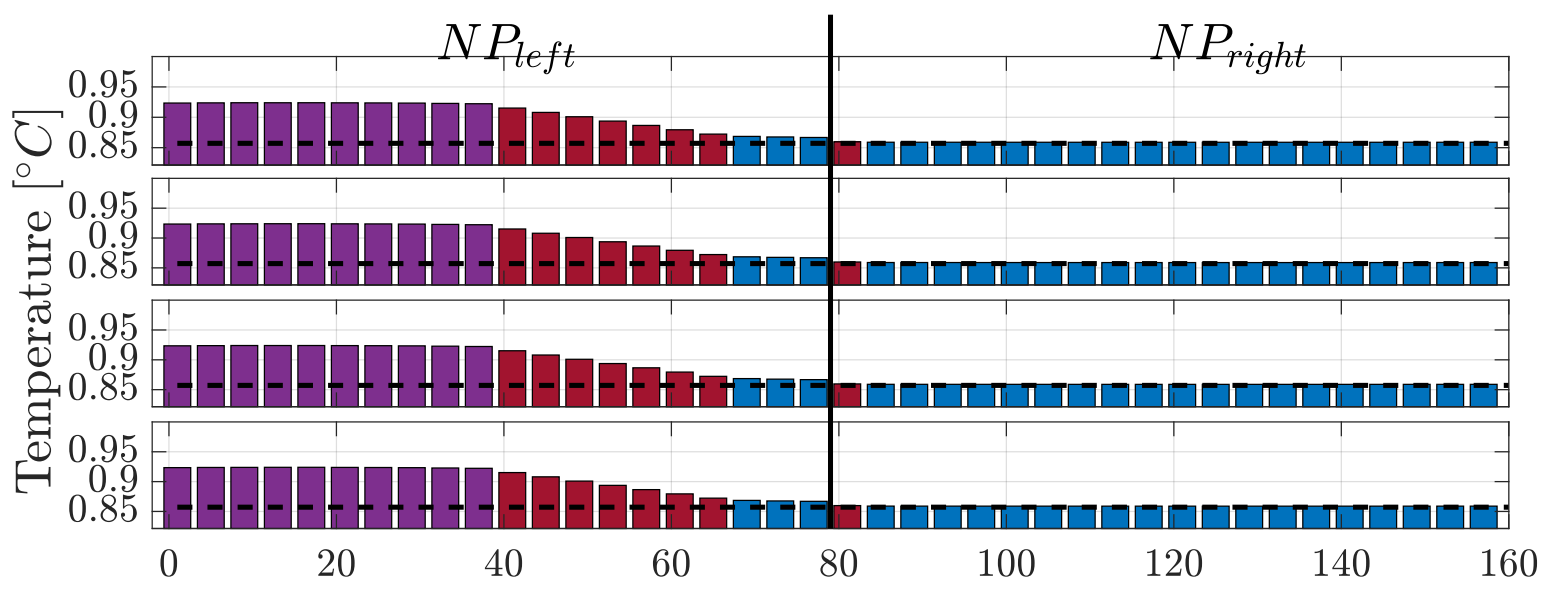

(a) Controlled Scenario: DM0 is fully used for jetting.

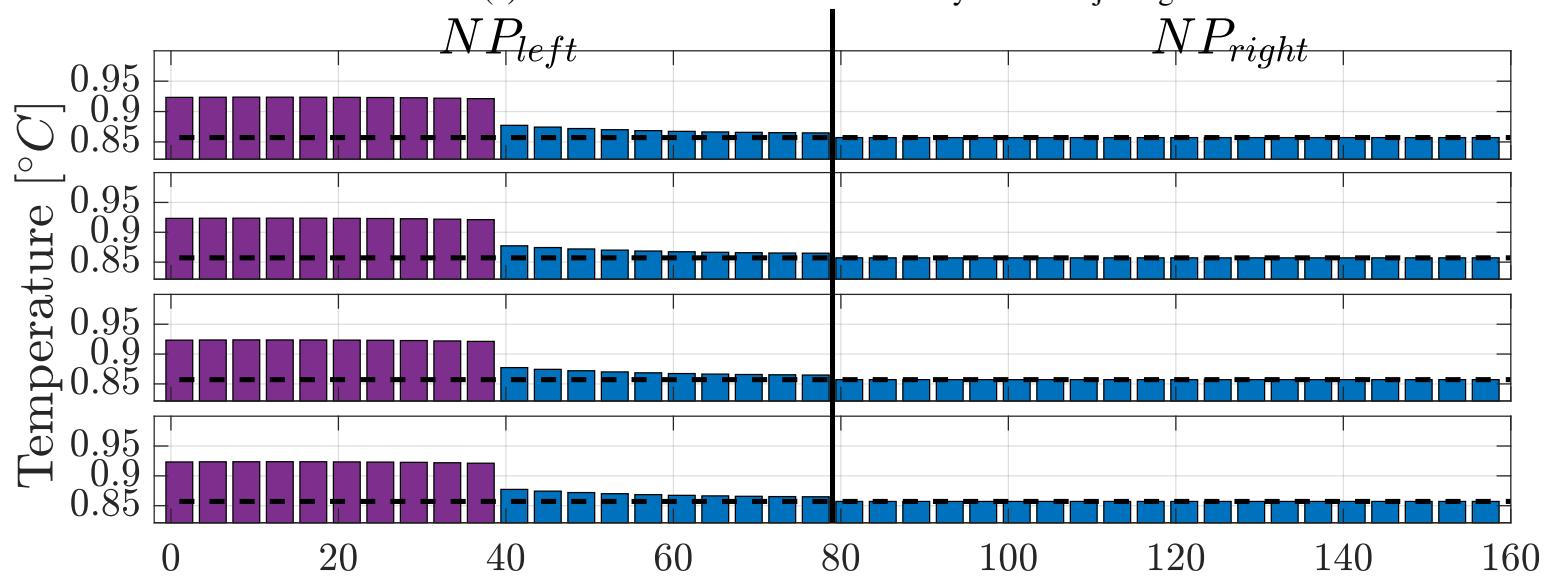

(b) Uncontrolled Scenario: DM0 is fully used for jetting.

Fig. 18: Up-scaled simulation for 160 nozzles. used as thermal actuators. denotes the nozzles that are not used for heating. Data points are scaled by a fixed constant.

minal constraint, the corresponding LMI (23) is solved using freely available YALMIP in MATLAB ${ }^{\circledR}$. Once the optimization yields the optimal control inputs, they are applied to the heating piezoelectric actuators by means of non-jetting voltage pulses. The MPC then repeats the same procedure over the entire print-job iteratively.

Remark VI.1. (On computational complexity) With increasing number of the nozzles, evidently, the computational complexity of solving MPC increases. At a specific iteration $k$, number of decision variables are related to the number of states, the number of control inputs and the number of horizons. Let they be $n_{x}, n_{h}^{k}$ and $N$ respectively. In contrast to solving (21)-(22) that has a complexity of $\mathcal{O}\left(N^{3}\left(n_{x}+n_{h}^{k}\right)^{3}\right)$, the dense LCQP (24) has a lower complexity $\mathcal{O}\left(N^{3} n_{h}^{k^{3}}\right)$ [23]. This is achieved by eliminating the states from the decision variables with substitution of equality constraints. However, in condensed formulation, the sparsity of matrices is partially lost [24]. Yet the dense LCQP (24) is favorable as the dimension of decision variables involves only the number of non-jetting heating actuators. This is typically small in number.
5) Results: Once the MPC is applied, the ink temperature of all 48 nozzles are shown in figure 17. It demonstrates that the performance specifications are met while satisfying the constraints. Moreover, only the adjacent heating nozzles are being used to compensate for the temperature inconsistencies among jetting and non-jetting nozzles.

Due to the modularity of the digital twin, up-scaling the number of nozzles does not require re-building the entire model. The digital twin and the control software are automated to build an up-scaled model with user-defined number of nozzles, implement in situ sensing-actuation scheme using the bit-map and visualize results of the closed-loop system once the MPC is applied. To demonstrate that, the same printhead, shown in figure 1a, is equipped with 160 nozzles that are equally divided over $N P_{\text {left }}$ and $N P_{\text {right }}$. In each side, there are 80 nozzles that are equally distributed over four rows. In figure 18, the result of MPC is shown when half the nozzles in $N P_{l e f t}$ are used for jetting.

\section{CONCLUSIONS AND FUTURE WORK}

In this paper, a modular and flexible digital twin is presented for modeling and control of thermo-fluidic processes 
in an inkjet printhead. In particular, no additional sensors or actuators are incorporated to develop an in situ sensingactuation based control strategy that minimizes the ink temperature fluctuations among individual nozzle. To this end, an experimentally validated graph-theoretic modeling framework is developed that is modular up to arbitrary number of nozzles. A control strategy was implemented without using additional sensors and without using additional actuators. In particular, to circumvent this limitation, the piezoelectric elements at every individual nozzles serves three roles: a) it is a jetting actuator for depositing liquid ink, b) it is a soft sensor for estimating ink temperature, and c) it is a control actuator to diminish gradient in ink temperature among nozzles. Once a voltage pulse is applied to a piezoelectric material, the soft-sensor uses its self-sensing mechanism to measure the acoustic signal. An algorithm is presented that uses the energy of this acoustic signal to estimate the ink temperature or ink viscosity at every individual nozzle. An MPC controller uses the estimated inktemperature to maintain the fluctuation of ink temperature among nozzles below a range of $\pm 0.3^{\circ} \mathrm{C}$ while using only non-jetting piezoelectric elements as thermal actuators.

Questions related to different control architecture, e.g. decentralized or distributed architecture for reducing signal overload and faster MPC computation are not addressed in this paper. Moreover, designing and scheduling voltage pulses for actuating (non-jetting) nozzles that apply required control input without forming droplets of ink are kept as future research.

\section{REFERENCES}

[1] S. W. Crompton, The Printing Press. Transforming Power of Technology, ser. Advance in Commercial Technology. Chelsea House Publishers, 2004, vol. 1.

[2] M. G. Wassink, "Inkjet printhead performance enhancement by feedforward input design based on two-port modeling," Ph.D. dissertation, Technische Universiteit Delft, Netherlands, February 2006.

[3] C. H. Séquin, "Rapid prototyping: A 3d visualization tool takes on sculpture and mathematical forms," Commun. ACM, vol. 48, no. 6 , pp. 66-73, Jun. 2005. [Online]. Available: http://doi.acm.org/10.1145/ 1064830.1064860

[4] A. Khalate, "Model-based feedforward control for inkjet printheads," Ph.D. dissertation, Technische Universiteit Delft, Netherlands, December 2013.

[5] W. Zapka, Handbook of Industrial Inkjet Printing: A Full System Approach. Volume 1 \& 2. Wiley-VCH Verlag gmbH \& Company KGaA, no. v. 2. [Online]. Available: https://books.google.nl/books?id= HXPswEACAAJ

[6] A. Fraters, "Inkjet printing: bubble entrainment and satellite formation," Ph.D. dissertation, University of Twente, Netherlands, 122018.

[7] D. B. Bogy and F. E. Talke, "Experimental and theoretical study of wave propagation phenomena in drop-on-demand ink jet devices," IBM J. Res. Dev., vol. 28, no. 3, pp. 314-321, May 1984. [Online]. Available: http://dx.doi.org/10.1147/rd.283.0314

[8] H. Wijshoff, "Structure and fluid dynamics in piezo inkjet printheads," Ph.D. dissertation, Technische Universiteit Delft, 12008.

[9] S. Koekebakker, M. Ezzeldin, A. Khalate, R. Babuška, X. Bombois, P. van den Bosch, G. Scorletti, S. Weiland, H. Wijshoff, R. Waarsing, and W. de Zeeuw, Piezo Printhead Control: Jetting Any Drop at Any Time. New York, NY: Springer New York, 2013, pp. 41-85. [Online]. Available: https://doi.org/10.1007/978-1-4614-4821-1_3

[10] J. H. Lienhard IV and J. H. Lienhard V, "A HEAT TRANSFER TEXTBOOK, fourth edition," Phlogiston Press, 2017.

[11] K. J. Kircher and K. M. Zhang, "On the lumped capacitance approximation accuracy in RC network building models," Energy and Buildings, vol. 108, pp. 454-462, 2015. [Online]. Available: http://www.sciencedirect.com/science/article/pii/S0378778815302930
[12] D. Yi and M. Zhang, "Heat flux investigations during flame thermal spray process using the lumped capacitance method," Applied Thermal Engineering, 2017.

[13] VDI Heat Atlas. Berlin, Heidelberg: Springer Berlin Heidelberg, 2010. [Online]. Available: http://dx.doi.org/10.1007/978-3-540-77877-6

[14] R. Tóth, M. Lovera, P. S. Heuberger, M. Corno, and P. M. Van den Hof, "On the discretization of linear fractional representations of lpv systems," IEEE Transactions on Control Systems Technology, vol. 20, no. 6, pp. 1473-1489, 2012.

[15] R. Ambur and S. Rinderknecht, "Self-sensing techniques of piezoelectric actuators in detecting unbalance faults in a rotating machine," Procedia Engineering, vol. 144, pp. 833 - 840, 2016, international Conference on Vibration Problems 2015. [Online]. Available: http: //www.sciencedirect.com/science/article/pii/S1877705816303125

[16] K. Kwon, "Waveform design methods for piezo inkjet dispensers based on measured meniscus motion," Journal of Microelectromechanical Systems, vol. 18, no. 5, pp. 1118-1125, Oct 2009.

[17] A. van der Bos, R. Jeurissen, M. Versluis, D. Lohse, H. Reinten, H. Wijshoff, M. van den Berg, and J. de Jong, "Acoustic measurement of bubble size and position in an ink jet printhead." The Journal of the Acoustical Society of America, 2013.

[18] J. F. Dijksman, "Hydrodynamics of small tubular pumps," 1984.

[19] H. Usuda, "Droplet discharging apparatus and method," 2004, patent no. US2004/0135832A1. [Online]. Available: http://www.freepatentsonline. com/y2004/0135832.html

[20] H. Nishimura, "Temperature uniformity across an inkjet head using piezoelectric actuation," 2017, patent no. EP3213918A1. [Online]. Available: https://patentimages.storage.googleapis.com/44/e3/ 87/46409e57a578aa/EP3213918A1.pdf

[21] U. Maeder, F. Borrelli, and M. Morari, "Linear offset-free model predictive control," Automatica, vol. 45, no. 10, pp. 2214 - 2222, 2009. [Online]. Available: http://www.sciencedirect.com/science/article/ pii/S0005109809002969

[22] D. Mayne, J. Rawlings, C. Rao, and P. Scokaert, "Constrained model predictive control: Stability and optimality," Automatica, vol. 36, no. 6, pp. 789 - 814, 2000. [Online]. Available: http://www.sciencedirect.com/science/article/pii/S0005109899002149

[23] J. Maciejowski, Predictive Control with Constraints. England.: Prentice Hall, 2002.

[24] J. L. Jerez, E. C. Kerrigan, and G. A. Constantinides, "A sparse and condensed qp formulation for predictive control of lti systems," Automatica, vol. 48, no. 5, pp. 999 - 1002, 2012. [Online]. Available: http://www.sciencedirect.com/science/article/pii/S0005109812001069 